\title{
Investigating the Projected Phase Space of Gaussian and Non-Gaussian Clusters
}

\author{
V. M. Sampaio, ${ }^{1,2 \star}$ R. R. de Carvalho,${ }^{2}$ I. Ferreras,,${ }^{3,4,5}$ T. F. Laganá, ${ }^{2}$
}

A. L. B. Ribeiro, ${ }^{6}$ S. B. Rembold ${ }^{7}$

${ }^{1}$ Divisão de Astrofísica (INPE-MCT), São José dos Campos, 12227-010, SP, Brazil

${ }^{2}$ NAT - Universidade Cruzeiro do Sul / Universidade Cidade de São Paulo, 01506-000, SP, Brazil

${ }^{3}$ Instituto de Astrofísica de Canarias, Calle Ví a Láctea s/n, E38205, La Laguna, Tenerife, Spain

${ }^{4}$ Department of Physics and Astronomy, University College London, Gower Street, London WC1E 6BT, UK

${ }^{5}$ Departamento de Astrofísica, Universidad de La Laguna, E38206 La Laguna, Tenerife, Spain

${ }^{6}$ Laboratório de Astrofísica Teórica e Observacional, Universidade Estadual de Santa Cruz, 45650-000, BA, Brazil

${ }^{7}$ Universidade Federal de Santa Maria, 97105-900, RS, Brazil

Accepted 2021 March 03. Received 2021 March 02; in original form 2020 December 01

\begin{abstract}
By way of the projected phase-space (PPS), we investigate the relation between galaxy properties and cluster environment in a subsample of groups from the Yang Catalog. The sample is split according to the gaussianity of the velocity distribution in the group into gaussian $(\mathrm{G})$ and non-gaussian (NG). Our sample is limited to massive clusters with $\mathrm{M}_{200} \geq 10{ }^{14} \mathrm{M}_{\odot}$ and $0.03 \leq \mathrm{z} \leq 0.1$. NG clusters are more massive, less concentrated and have an excess of faint galaxies compared to $\mathrm{G}$ clusters. NG clusters show mixed distributions of galaxy properties in the PPS compared to the G case. Using the relation between infall time and locus on the PPS, we find that, on average, NG clusters accreted $\sim 10^{11} \mathrm{M}_{\odot}$ more stellar mass in the last $\sim 5$ Gyr than G clusters. The relation between galaxy properties and infall time is significantly different for galaxies in $\mathrm{G}$ and NG systems. The more mixed distribution in the PPS of NG clusters translates into shallower relations with infall time. Faint galaxies whose first crossing of the cluster virial radius happened 2-4 Gyr ago in NG clusters are older and more metal-rich than in $\mathrm{G}$ systems. All these results suggest that NG clusters experience a higher accretion of pre-processed galaxies, which characterizes $\mathrm{G}$ and NG clusters as different environments to study galaxy evolution.
\end{abstract}

Key words: galaxies: clusters: general - evolution - galaxies: formation

\section{INTRODUCTION}

The study of galaxies in the nearby Universe reveals that their evolution is fundamentally dependent on their environment. From the core to the outskirts of clusters, the galactic density roughly spans seven orders of magnitude, and the morphology-density relation established by Dressler (1980) is one of the simplest examples of how the environment affects galaxy properties. Early-type galaxies (ETGs) inhabit the core (high-density regime), while Late-type galaxies (LTGs) preferentially populate the outskirts (i.e. at lower densities). In the last decades, observations show that galaxies usually evolve from star-forming late-type systems to passive/quiescent early-type ones. These two extreme cases explain the bimodality observed, for instance, in star-formation rate (SFR, e.g. Wetzel et al. 2012a) and in gas content. The dichotomy between star-forming LTGs and quiescent ETGs in the stellar mass versus SFR defines the so-called Blue Cloud (BC) and Red Sequence (RS), respectively.

^ E-mail: vitorms999@gmail.com
An intermediate region, Green Valley $(\mathrm{GV})$, reinforces the idea of having physical mechanisms that quench star formation transforming a system in the BC into one in the RS (e.g. Angthopo et al. 2019). In clusters, galaxies lose their galactic hot gas as they fall (depending on their total mass), even before entering the cluster virial region (through strangulation/starvation, Larson et al. 1980; Balogh et al. 2000; van de Voort et al. 2017). After crossing the virial region, gravitational tides from the cluster deep potential well strip away the interstellar medium, stars and dark matter from the infalling galaxy (tidal stripping, Johnston et al. 1999; Read et al. 2006). Furthermore, the hot gas in the intra-cluster medium (ICM) exerts pressure on galaxies moving within the cluster and may remove gas via ram pressure stripping (RPS, Gunn \& Gott 1972; Abadi et al. 1999). Galaxy clusters also provide a suitable environment for galaxy-galaxy interactions, especially in the core. Indirect continuous encounters between galaxies within the cluster may leave interacting galaxies with distorted features. Direct encounters may lead to galaxy mergers, and cause a burst in star formation over a short time scale and rapidly exhaust the gas component (Springel \& 
Hernquist 2005; Cox et al. 2008; Teyssier et al. 2010). In addition, previous episodes inhabiting groups with lower halo mass can alter the properties of galaxies even before infalling into clusters, an effect called pre-processing (Fujita 2004; Mahajan 2013; Sarron et al. 2019). There are also internal feedback processes driven by AGN, supernovae and stellar winds that cause gas outflows that diminish the gas reservoir of galaxies (mass quenching, Larson 1974; Dekel \& Silk 1986; Bongiorno et al. 2016).

The variety of mechanisms quenching star formation fuels the debate concerning the most important driver, and the related time scales. Trussler et al. (2020) study galaxies in the local Universe and suggest that quenching has an extended phase ( $\sim 5 \mathrm{Gyr})$ of starvation. However, several studies find that the main quenching mechanism is dependent on halo mass ( $\mathrm{Zu} \&$ Mandelbaum 2016). Peng et al. (2010) show that a stellar mass related mechanism plays a major role in quenching massive galaxies. Trussler et al. (2020) show also that gas outflows are increasingly relevant at decreasing stellar mass. In addition, Rhee et al. (2017) (R17, hereafter) suggest that galaxies in clusters lose a significant fraction of their mass over long time scales due to tidal mass loss (TML, see their Fig. 3). On the other hand, in short time-scales ( 2 Gyr), RPS is found to be the main quenching mechanism, but only above a density threshold (Roberts et al. 2019, R19 hereafter). The cumulative effect of different quenching mechanisms acting simultaneously in galaxies results in a non-linearity of the general quenching process. Several models have been proposed in the last decades to simplify this inherent non-linearity. One of the most recently adopted hypothesis is the so-called "delayed-then-rapid" quenching model (Wetzel et al. 2013). In this model, an infalling galaxy is unaffected by the cluster environment for a delay time after which it becomes a satellite and is mostly quenched due to starvation in this phase. After the delay time, the cluster environment strongly affects star formation, that is rapidly quenched due to RPS.

Star formation quenching is also conditional on cluster properties. For instance, RPS depends on the ICM density and its velocity relative to the galaxy. In order to characterize different environments, substructure analyses in the optical (e.g. Dressler \& Shectman 1988; Girardi et al. 1997) and X-ray (e.g. Schuecker et al. 2001; Zhang et al. 2009) indicate that many clusters are not fully virialized. The degree of relaxation is also related to the orbital parameters. Infalling galaxies have highly radial orbits in the outskirts, while virialized objects show circular orbits within the virial radius. It follows from statistical mechanics that the equilibrium state of a dynamical system may be well described by a defined velocity distribution. Ogorodnikov (1957) and Lynden-Bell (1967) suggest a Maxwell-Boltzmann distribution in velocity as an equilibrium state for gravitationally bound systems. This result, however, rests on simplifying assumptions that may be unrealistic (clusters evolving in isolation and gravitation as the only interaction, for example). An additional limitation is that the observations are projected along the line-of-sight (LOS). The use of N-body simulations gives support to the adoption of a Maxwell-Boltzmann distribution, that leads to a Gaussian distribution in velocity projected along the line of sight (Merrall \& Henriksen 2003; Hansen et al. 2005). Previous studies investigate the difference between clusters with projected velocity distributions well-fit by a Gaussian $(G)$ and those with a non-Gaussian (NG) velocity profile and find that: 1) NG clusters have an excess of star-forming galaxies (Ribeiro et al. 2010); 2) the stellar population parameters of infalling and virialized galaxies in NG clusters are not well separated as in G clusters (Ribeiro et al. 2013); 3) there is evidence of a higher infall rate of pre-processed galaxies in NG clusters (Roberts \& Parker 2017; de Carvalho et al.
$2017)$; 4) the velocity dispersion profiles of cluster members is significantly different between $\mathrm{G}$ and NG systems (Costa et al. 2018); and 5) simulations show that NG systems suffered their last major merger more recently than G systems (Roberts \& Parker 2019). These results point towards a higher infall rate in NG clusters in comparison to $\mathrm{G}$ clusters. However, the separation between $\mathrm{G}$ and NG clusters rely on a robust measure for gaussianity. AndersonDarling test (Anderson \& Darling 1952) and Gaussian Mixture Models (Reynolds 2009) are among the most employed methods to measure gaussianity. However, comparing two general distributions is a longstanding problem in statistics. For instance, several methods implement the testing of the null hypothesis that two populations corresponding to different datasets originate from the same parent distribution (e.g. Mann-Whitney-Wilcoxon, Hodges-Lehmann, Kruskal-Wallis, etc.) but none yields a definite answer to the problem (Feigelson \& Babu 2012). In this particular case, the aim is for a clear, quantitative and unambiguous distinction between the velocity distributions into $\mathrm{G}$ and $\mathrm{NG}$.

As an option to study the velocity distribution, the Lagrangian formulation of motion enables the study of the dynamical evolution of physical systems through the "phase-space", which combines both spatial and velocity coordinates into a single space. In clusters, this complex 6D space is simplified to a diagram comprising cluster-centric distance and velocity. Galaxies infalling in clusters have a well-defined trajectory in phase-space (see Fig. 1 of R17). Nevertheless, observations are limited to projected quantities and so phase space is constrained to a projected version, the so-called projected phase space (PPS, hereafter). In the PPS, the aforementioned $\mathrm{G}$ or NG velocity distributions are simply a projection along the y-axis. The PPS is thus a more informative space than velocity distribution alone. It is also important to emphasize that environmental effects, such as RPS, are conditional on the velocity of the infalling galaxy and thus the PPS provides a more suitable way to study environmental effects. Oman et al. (2020) combine data from the SDSS to show that galaxies take around $\sim 3 \mathrm{Gyr}$ after the first pericentric passage to be quenched. Although the well-defined trajectory in phase space becomes degenerate in the PPS due to projection effects, Mahajan et al. (2011) and Oman et al. (2013) use numerical simulations and suggest that galaxies at different orbits - and consequently in different dynamical stages inside the cluster - occupy distinct regions in the PPS. Pasquali et al. (2019) (hereon P19) study the distribution of infall time $\left(\mathrm{t}_{\text {inf }}\right)$ - defined as the time duration from when the galaxy reached the virial radius of the main progenitor of its present-day host environment, for the first time - in the PPS and define regions that constrain galaxies within a narrow width of $t_{\text {inf }}$. R17 investigate the relation between tidal mass loss, $\mathrm{t}_{\mathrm{inf}}$ and the region occupied in the PPS. Finally, Rhee et al. (2020) propose a relation between SFR and time since infall based on the region occupied in the PPS.

In this work, we follow a methodology that focuses on the PPS to investigate further differences between $\mathrm{G}$ and NG clusters. We map the PPS with a grid and explore the properties in each galactic environment. Taking stellar mass as a key parameter, we derive a rough estimate of the infall rate in NG clusters from the observed distribution. We perform a statistical study in the different regions and evaluate the global properties of the PPS of G and NG clusters. We also build a relation between galaxy properties and infall time using PPS regions presented in P19.

This paper is organized as follows: in $\S 2$ we define the sample, present the stellar population parameters and dynamical properties of the galaxies, and describe the related methods. In $\S 3$ we present a first characterization of the structure of $\mathrm{G}$ and NG clusters and 
their galactic content. $\S 4$ introduces the methods adopted to build the PPS and define different loci of interest. In $\S 5$ we connect galaxy properties with their position on the PPS diagram. In $\S 6$ we explore the relation between time since infall and stellar population parameters. In $\S 7$ we derive a first estimate of the infall rate in NG clusters with respect to $\mathrm{G}$ clusters. Finally, in $\S 8$ we discuss the relationship between the gaussianity of the velocity distribution and the properties of cluster members. This paper adopts a flat $\Lambda \mathrm{CDM}$ cosmology with $\left[\Omega_{\mathrm{M}}, \Omega_{\Lambda}, \mathrm{H}_{0}\right]=\left[0.27,0.73,72 \mathrm{~km} \mathrm{~s}^{-1} \mathrm{Mpc}^{-1}\right]$.

\section{SAMPLE AND DATA}

Our sample is based on the Yang Catalog (Yang et al. 2007), which uses a halo finder algorithm applied to the New York University Value-Added Galaxy Catalog (NYU - VAGC, Blanton et al. 2005). The catalog was originally based on the fifth data release of the Sloan Digital Sky Survey (SDSS-DR5, Adelman-McCarthy et al. 2007). We make use of an updated version, presented in de Carvalho et al. (2017) (dC17, hereafter), based on the seventh data release (SDSS-DR7).

\subsection{Data Selection and Membership Assignment}

We use similar data to those presented in $\mathrm{dC} 17$ and Costa et al. (2018). We select SDSS-DR7 galaxies with line-of-sight velocities in the range $\pm 4,000 \mathrm{~km} \mathrm{~s}^{-1}$ and a projected distance $\mathrm{d}_{\text {proj }} \leq$ 2.5 $\mathrm{h}^{-1} \mathrm{Mpc}$ (i.e. $3.47 \mathrm{Mpc}$ for $\mathrm{h}=0.72$ ) from the clustercentric coordinates described in the Yang Catalog. We use galaxies in the redshift interval $0.03 \leq \mathrm{z} \leq 0.1$, and $\mathrm{r}$-band magnitudes $\mathrm{m}_{\mathrm{r}} \leq 17.78$, which is the survey spectroscopic completeness limit. These criteria guarantee that we probe the luminosity function to $M^{\star}+1$ mag. The lower limit in redshift avoids large aperture-related bias in the stellar population parameters due to the fixed 3 arcsec diameter of the SDSS fibers.

Galaxy membership is defined via an iterative Shiftgapper technique (see Lopes et al. 2009b). Next we briefly describe how this technique works. First, we select SDSS-DR7 galaxies around the cluster center presented in the Yang Catalog ${ }^{1}$ to feed the Shiftgapper technique. Then the algorithm follows the methodology presented in Fadda et al. (1996). Namely, we apply a gap technique in radial bins with sizes $0.43 \mathrm{~h}^{-1} \mathrm{Mpc}^{2}$ and remove galaxies with a velocity gap greater than $1000 \mathrm{~km} \mathrm{~s}^{-1}$ relative to the mean cluster velocity (see Fig. 1 in Fadda et al. 1996). This procedure is reiterated until there are no more interlopers. We define the clustercentric coordinates as the luminosity weighted RA, DEC and median redshift. We use this technique to redefine the Yang catalog due to two main reasons: 1) Shiftgapper avoids prior assumptions about the dynamical state of the system; and 2) comparison shows that Shiftgapper is less restrictive than the Halo mass finder algorithm, which is relevant to works investigating satellite galaxy properties. We perform a virial analysis (see Lopes et al. 2009a) in the final list of members to estimate dynamical quantities like virial radius $\left(\mathrm{R}_{200}\right)$, virial mass $\left(\mathrm{M}_{200}\right)$, and velocity dispersion along the line of sight $\left(\sigma_{\mathrm{LOS}}\right)$. We highlight that the Shiftgapper method returns a list of galaxies without interlopers, which will be relevant in Section 7. Finally, we restrain our sample to systems with at least 20 galaxy

1 We highlight this is the only use of the Yang Catalog

2 This bin size ensures at least 15 galaxies in each bin members within $\mathrm{R}_{200}$ (see Section 2.2 for this threshold explanation), resulting in 319 systems. We separate member galaxies into two different luminosity regimes: 1 ) Bright (B): $0.03 \leq z \leq 0.1$ and $\mathrm{M}_{\mathrm{r}} \leq-20.55 \sim \mathrm{M}^{\star}+1 \mathrm{mag}$, where $\mathrm{M}_{\mathrm{r}}$ is the limiting absolute magnitude in the r-band; and 2) Faint (F): $0.03 \leq z \leq 0.04$ and $-20.55<\mathrm{M}_{\mathrm{r}} \leq-18.40 \sim \mathrm{M}^{\star}+3$ mag. The redshift upper limit in the Faint sample corresponds to the spectroscopic completeness limit for $\mathrm{M}_{\mathrm{r}}=-18.40$ in the SDSS.

\subsection{Characterizing Different Galactic Environments}

We characterize the cluster environment through the gaussianity of the projected velocity distribution. Comparing and classifying distributions is a long-standing problem in statistics due to the difficulty on measuring the distance between two distributions (De Helguero 1904, Schilling et al. 2002). For simplicity, we may assume that multimodal expression patterns results from multiple interacting groups; bimodality expresses two groups in interaction or a perturbation of a single Gaussian distribution; and unimodality represents a system close to virialization. The problem is then how to find multiple modes (Gaussians for instance) in a distribution. Thus, we can either identify a certain mixture of multiple modes (Gaussians) justifying the observed distribution or we determine how far from a Gaussian a distribution is. Each approach has its pros and cons. $\mathrm{dC} 17$ tackle the problem by creating realizations representing a perfect mixture of Gaussians and show that the later method (measuring the distance from a Gaussian) works better. This simplification, although not representing what happens in real clusters, serves as a guide to study multimodality modeling.

In this work, we make use of the classification method presented in $\mathrm{dC} 17$, based on the Hellinger Distance (HD), that measures the distance between two discrete distributions, $\mathrm{P}_{1}$ and $\mathrm{P}_{2}$, and is expressed as:

$\mathrm{HD}^{2}\left(\mathrm{P}_{1}, \mathrm{P}_{2}\right)=2 \sum_{\mathrm{x}}\left[\sqrt{\mathrm{p}_{1}(\mathrm{x})}-\sqrt{\mathrm{p}_{2}(\mathrm{x})}\right]^{2}$,

where $\mathrm{p}_{1}$ and $\mathrm{p}_{2}$ are the two probability density functions (PDFs) and $x$ is a random variable (see Le Cam \& Yang 2012, for more details).

A detailed HD characterization and the threshold between Gaussian and NG velocity distribution as a function of members galaxies number is presented in $\mathrm{dC} 17$. In a nutshell, $\mathrm{dC} 17$ probe the parameter space defining a bimodal distribution and find which parameter mostly affects the distance between the two distributions. The HD proved to be robust in distributions with at least 20 members within $R_{200}$, which translates to a mass cutoff. The relation between $\mathrm{M}_{200}$ and $\mathrm{N}_{200}$ (where $\mathrm{N}_{200}$ is the number of B galaxies inside $\mathrm{R}_{200}$ ) yields a lower mass threshold of $10^{14} \mathrm{M}_{\odot}$ for our sample. We also restrict our sample to systems with at least $70 \%$ reliability, measured with a bootstrap technique, on the gaussianity classification. This reduces the sample from 319 to 177 clusters (split into $143 \mathrm{G}$ and $34 \mathrm{NG}$ ). The ratio of $\mathrm{G}$ and NG clusters in our sample (G/NG $\sim 81 \%$ ) is in agreement with previous works (e.g. Ribeiro et al. 2013). Our final sample comprises 6,578 B galaxies $(4,817$ in $\mathrm{G}$ clusters and 1,661 in NG) and 2,205 F galaxies (907 in $\mathrm{G}$ and 1,298 in NG).

\subsection{Derived Stellar Population Parameters}

We select age, stellar metallicity $([\mathrm{Z} / \mathrm{H}])$ and stellar mass $\left(\mathrm{M}_{\text {stellar }}\right)$ from the $\mathrm{dC} 17$ galaxy Catalog to characterize the stellar population content. The estimates are derived from full spectral fitting 
Table 1. Uncertainties in stellar population parameters.

\begin{tabular}{c|ccc}
\hline$\Delta$ & Age $(\mathrm{Gyr})$ & {$[\mathrm{Z} / \mathrm{H}](\mathrm{dex})$} & $\log \left(\mathrm{M}_{\text {stellar }} / \mathrm{M}_{\odot}\right)(\mathrm{dex})$ \\
\hline BG & $0.18 \pm 0.35$ & $0.003 \pm 0.019$ & $0.01 \pm 0.01$ \\
FG & $0.26 \pm 0.61$ & $0.006 \pm 0.032$ & $0.06 \pm 0.03$ \\
\hline
\end{tabular}

using the STARLIGHT code (Cid Fernandes et al. 2005), that fits the input galaxy spectra with a superposition of pre-defined single stellar populations (SSP). The age and stellar metallicity are estimated from the weighted sum of the combination of SSP parameters that gives the best fit, and are only derived for spectra without any anomalies (see $\mathrm{dC} 17$ for more details). The adopted stellar models are based on the Medium resolution INT Library of Empirical Spectra (MILES, Sánchez-Blázquez et al. 2006), that features an almost constant spectral resolution $(\sim 2.5 \AA)$. The SSP basis grid has a constant $\log$ (Age) steps of 0.2 dex from 0.07 to $14.2 \mathrm{Gyr}$ and includes SSPs with $[\mathrm{Z} / \mathrm{H}]=\{-1.71,-0.71,-0.38,0.00,+0.20\} . \mathrm{M}_{\text {stellar }}$ is derived within the fiber and then extrapolated to the whole galaxy by computing the difference between fiber and model magnitudes in the $z$-band (Trevisan et al. 2012), assuming no gradients in the population content. Therefore, $\mathbf{M}_{\text {stellar }}$ is given by:

$\log \left(\mathrm{M}_{\text {stellar }}\right)=\log \left(\mathrm{M}_{\text {stellar }}\right)^{\prime}+0.4\left(\mathrm{~m}_{\text {fiber }, \mathrm{z}}-\mathrm{m}_{\text {model }, \mathrm{z}}\right)$.

Spectral fitting codes allow arbitrary weighting and there are two commonly adopted methods in the literature: 1) luminosityweighted parameters trace mainly younger stellar population properties; while 2) mass-weighted parameters are more closely related to the cumulative galaxy evolution (see, Trussler et al. 2020). In the following, we use luminosity-weighted parameters, less prone to biases. In this case, age will be closely related to the last episode of star formation.

\subsection{Assessing Derived Stellar Population Uncertainties}

The stellar population parameters have an intrinsic uncertainty as any derived quantity. Part of this uncertainty comes from the observed spectra, which can vary over different observations. To tackle this, we use a subsample of SDSS-DR7 galaxies with repeated observations. This set covers the same redshift and magnitude range of our main sample and is limited to observations with a signalto-noise ratio greater than 20 in the $r$ band. This results in 6,148 observations of 2,543 galaxies. We calculate the uncertainty separately for the two luminosity regimes, bright (B) and faint $(\mathrm{F})$. A direct comparison yields the residuals shown in Table 1. Column (1) shows the luminosity regime considered for the residuals; columns (2), (3) and (4) list the residuals and the errors in Age, $[\mathrm{Z} / \mathrm{H}]$ and $\mathrm{M}_{\text {stellar, }}$, respectively. As expected, the stellar population parameters of faint galaxies have higher uncertainties than bright galaxies. From here on we adopt the measurement errors shown in Table 1. We compare these measurement errors with statistical uncertainties where it is relevant.

\subsection{Additional Galaxy Properties}

In addition to the stellar population parameters, we retrieve star formation rates (SFR), morphology and color gradients, further tracers of galaxy evolution. In this subsection, we describe relevant galaxy properties retrieved from other works than $\mathrm{dC} 17$.

\subsubsection{SDSS-DR13 Spectroscopic Information}

We retrieve Star Formation Rates (SFR) from the MPA-JHU cata$\log$, that provides measurements for all SDSS-DR13 galaxies with reliable spectra (Albareti et al. 2017). The available SFRs were computed following Brinchmann et al. (2004), that use the $\mathrm{H}_{\alpha}$ line luminosity measured within the spectroscopic fiber, correcting the aperture effect with photometry. Our query of the SDSS-DR13 database results in SFR estimates for all galaxies, except for a set of 384 galaxies, which constitute a small percentage $(\sim 2.46 \%)$ of the total sample. We discard these galaxies only in the SFR-dependent analysis.

\subsubsection{Morphological Characterization}

Galaxy morphology is intimately related to stellar population parameters and thus galaxy evolution (see, e.g., Roberts \& Haynes 1994). Here we use the TType parameter as a tracer of galaxy morphology. It was first introduced by de Vaucouleurs (1963) to classify lenticular (S0) galaxies. Each galaxy is assigned a number based on morphological visual classification. Elliptical-like morphologies are denoted by TType $<0$, while TType $>0$ represents disk galaxies.

We select TType estimates from the Domínguez Sánchez et al. (2018) catalog, which uses deep learning algorithms based on Convolutional Neural Networks (CNN) to classify the morphology of 670,722 SDSS galaxies. TType classifications are in the range $[-3,10]$. Similar to SFR, this parameter is not available for all galaxies in our sample and we discard non-classified galaxies only in the TType dependent analysis. Statistically, this corresponds to a minor percentage of the whole sample, $\sim 1.33 \%$ (i.e 204 galaxies).

\subsubsection{Information from the Korea Institute for Advanced Study Value-Added Galaxy Catalog}

In our work, we add color gradient estimates from the Korea Institute for Advanced Study Value-Added Galaxy Catalog (KIASVAGC, Choi et al. 2010). The KIAS-VAGC catalog provides galaxy spectroscopic and morphological information for 593, 514 galaxies from the SDSS-DR7 main galaxy catalog and 10, 497 from other galaxy catalogs (see Park \& Choi 2005). The color gradient is computed using the color indices in the $\mathrm{g}$ and $\mathrm{i}$ bands. The $\mathrm{g}-\mathrm{i}$ color gradient is derived as:

$\nabla(\mathrm{g}-\mathrm{i})=(\mathrm{g}-\mathrm{i})_{0.5 \mathrm{R}_{\mathrm{p}}<\mathrm{r}<\mathrm{R}_{\mathrm{p}}}-(\mathrm{g}-\mathrm{i})_{\mathrm{r}<0.5 \mathrm{R}_{\mathrm{p}}}$,

where $(g-i)_{x}$ denotes the $g-i$ index where the condition $x$ is satisfied, and $R_{p}$ is the Petrosian Radius in the i-band. Equation 3 implies that more negative values correspond to bluer colors in the galaxy outskirts.

We use a 1.5 arcsec threshold in a positional cross-match between the KIAS-VAGC and our sample to select the appropriate estimates. The upper limit is defined empirically. We do not find reliable estimates for 304 galaxies, representing $1.96 \%$ of our sample. As in the previous cases, we discard non-classified galaxies only in the analysis pertaining to color gradients.

\section{STRUCTURE AND COMPOSITION OF G AND NG CLUSTERS}

In this work, the difference between G and NG clusters plays a major role. In a first step, we focus our attention on characterizing the structure and distribution of galaxy member properties in each 
class. In Fig. 1 we show the distributions of: a) logarithmic virial mass $\left(\log \left(\mathrm{M}_{200} / \mathrm{M}_{\odot}\right)\right)$; b) r-band absolute magnitude; c) a proxy for the concentration of stellar mass in each cluster, defined as $\mathrm{R}_{80} / \mathrm{R}_{20}$, where $\mathrm{R}_{\mathrm{X}}$ is the projected radius within which the stellar mass represents $x \%$ of the total stellar mass within $R_{200}$; d) velocity dispersion along the line-of-sight; and e) the distribution of cluster mean stellar mass of bright galaxies within $\mathrm{R}_{200}\left(\left\langle\mathrm{M}_{\text {stellar }}^{\mathrm{C}}\right\rangle\right)^{3}$ We compare the distributions using two different statistical tests: Anderson-Darling (AD) and Wilcoxon Rank Test (Wlx) (see Engmann \& Cousineau 2011 and Gehan 1965 for a review of both) ${ }^{4}$. The results are shown in each panel. Aside from the histograms, we add a kernel smoothed curve, shown as shaded area, which is derived directly from the dataset using an Epanechnikov Kernel Density estimator (Silverman 1986) with a bandwidth equal to 1.5 times the bin size. We find that $\mathrm{G}$ and NG clusters have statistically different distributions. In panel (a), we note that NG clusters tend to have higher values of $\mathrm{M}_{200}$ in comparison to G clusters. Namely, we find that $32.4 \%(11 / 34)$ of $\mathrm{NG}$ clusters have $\log \left(\mathrm{M}_{200} / \mathrm{M}_{\odot}\right)>14.75$, while this fraction decreases to $6.3 \%(9 / 143)$ in G clusters. The majority of $\mathrm{G}$ systems $(\sim 71.3 \%)$ have $\log \left(\mathrm{M}_{200} / \mathrm{M}_{\odot}\right)<14.5$. Panel (b) shows an excess of fainter galaxies 5 in NG clusters in comparison to $\mathrm{G}$ systems. We find that $30.5 \%$ of NG cluster members have $\mathrm{M}_{\mathrm{r}} \geq-19.5$, while the equivalent cut in absolute magnitude yields $14.1 \%$ of galaxies in $\mathrm{G}$ systems. Panel (c) shows the distribution of concentration in $\mathrm{G}$ and NG clusters, revealing that NG cluster galaxies are less concentrated than their $\mathrm{G}$ cluster counterparts. Only one NG cluster reaches $C>4$.7. In panel (d), we observe an excess of NG clusters with higher velocity dispersion in comparison to $\mathrm{G}$ clusters. NG clusters are presumed to be found in a non-virialized state, so that the expected velocity dispersions are higher, and, possibly, the estimated mass may also be an overestimate of the real cluster mass. In any case, this is one more piece of evidence regarding the different state of NG clusters with respect to G systems. Finally, we note in panel (f) an excess of NG clusters with mean stellar mass $3 \leq\left\langle\mathrm{M}_{\text {stellar }}^{\mathrm{C}}\right\rangle<4 \times 10^{11} \mathrm{M}_{\odot}$. In other words, we find that NG clusters have higher virial mass and radii, an excess of fainter galaxies, are less concentrated, contain more massive $\mathrm{B}$ galaxies and have higher velocity dispersion in comparison to $\mathrm{G}$ systems.

We give special attention to the mass mismatch of $\mathrm{G}$ and $\mathrm{NG}$ clusters. The excess of massive NG clusters is very likely related to the recent merger history of such systems. Roberts \& Parker (2019) use simulations to investigate the dynamical history of $\mathrm{G}$ and NG clusters and find that NG clusters suffered their last major merger more recently. Our results suggest that the difference in mass between $\mathrm{G}$ and $\mathrm{NG}$ likely follow from recent accretion. Additionally, Wetzel et al. (2012b) show that galaxy properties depend on halo mass, which can also be related to the separation between $\mathrm{G}$ and NG clusters. An usual approach to avoid mass bias is to compare mass-matched samples. However, we lack $\mathrm{G}$ clusters in the faint regime with $\log \left(\mathrm{M}_{200} / \mathrm{M}_{\odot}\right)>14.6$, which prevents the analysis of such a mass-matched sample in this regime. Namely, a massmatched sample in the faint regime would contain only 5 clusters. Nevertheless, we build a mass-matched sample in the bright regime and compare the results with the unmatched ones. Qualitatively, we find that our results do not depend on the use of a mass-matched

\footnotetext{
3 We consider only bright galaxies to avoid bias due to faint galaxies in clusters with $\mathrm{z}>0.04$

4 The adopted significance level threshold is $\alpha=0.05$

5 Not to be confused with the faint regime defined in Section 2.1
}
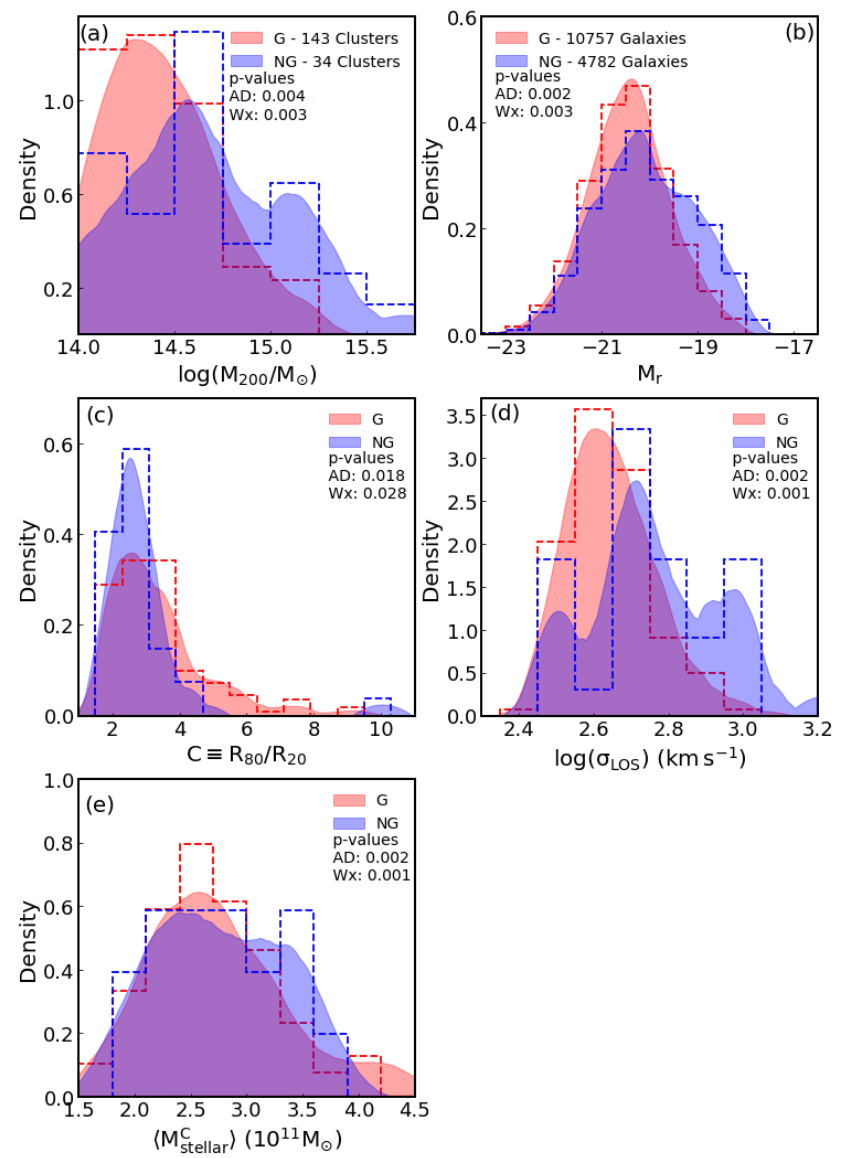

Figure 1. Comparison of the $\mathrm{M}_{200}$ (top-left), $\mathrm{M}_{\mathrm{r}}$ (top-right), $\mathrm{C} \equiv \mathrm{R}_{80} / \mathrm{R}_{20}$ (center-left), $\log \left(\sigma_{\text {LOS }}\right)$ (center-right) and cumulative stellar mass $\left(\mathrm{M}_{\text {stellar }}^{\mathrm{C}}\right)$ distribution according to the gaussianity classification ( $\mathrm{G}$ or NG cluster). In each plot we show the resulting p-value of the Anderson-Darling (AD) and Wilcoxon (Wx) statistical tests. NG clusters are more massive, less concentrated (see text for the concentration measurement adopted here), larger virial radius and have an excess of fainter galaxies with respect to $G$ systems.

sample. We present in Section 6 how the mass mismatch affects our results quantitatively.

Regarding galaxy properties, we compare the distribution of age, $[\mathrm{Z} / \mathrm{H}], \mathrm{M}_{\text {stellar }}, \mathrm{SFR}$, TType and color gradient $(\nabla(\mathrm{g}-\mathrm{i}))$ of $\mathrm{G}$ and NG cluster members in Figs. 2 (B galaxies) and 3 (F galaxies). In the bright case, we notice that $\mathrm{G}$ and NG clusters have statistically different distributions of age, TType and $\nabla(g-i)$. In panel (a) of Fig. 2, we notice in $\mathrm{G}$ clusters an excess of B galaxies with age $>7.5 \mathrm{Gyr}$ in comparison to NG systems. Regarding galaxies with age $<7.5 \mathrm{Gyr}$, the excess is seen in NG systems. In panels (b), (c) and (d), we note that $\mathrm{G}$ and NG clusters have a similar distribution of $[\mathrm{Z} / \mathrm{H}], \mathrm{M}_{\text {stellar }}$ and SFR. In panel (e), NG clusters feature an excess of B galaxies with TType $>3$, which translates to an excess of B galaxies with disk-like morphology. Finally, although the color gradient distributions (panel f) do not show great differences visually, statistical tests indicate significant differences between $G$ and NG clusters. Namely, we find a slight excess of galaxies with steeper color gradients in NG clusters. Extending the same analysis to F galaxies, shown in Fig. 3, we find that $\mathrm{M}_{\text {stellar }}$ is the only parameter to show statistically different distributions. We note a slight excess of faint galaxies with $\log \left(\mathrm{M}_{\text {stellar }} / \mathrm{M}_{\odot}\right)>10.2$ in $\mathrm{G}$ clusters 
in comparison to NG clusters. In all other panels we notice that the p-value analysis is unable to reject the null hypothesis that both distributions represent subsamples of the same parent distribution.

Following previous works (Roberts \& Parker 2017; de Carvalho et al. 2019, dC17), we would expect differences between $\mathrm{G}$ and NG cluster members mainly in the faint regime. However, the comparison of global distributions of $\mathrm{F}$ galaxy properties shows small (if any) differences. Furthermore, we find a different systematic in comparison to $\mathrm{dC} 17$, which may result from the use of the distribution itself instead of the cumulative distributions. Therefore, we use the PPS to better disentangle the expected differences.

\section{THE PROJECTED PHASE SPACE}

In this section, we describe how the PPS is defined in G and NG clusters, and contrast the results. The PPS diagram consists of two projected dynamical quantities: the peculiar velocity - measured along the line of sight $\left(\mathrm{V}_{\mathrm{LOS}}\right)$ - and the cluster-centric distance projected on the observer's plane $\left(\mathrm{R}_{\mathrm{LOS}}\right)$. We discard sign differences in the velocities (approaching/receding) and take the absolute value of $\mathrm{V}_{\mathrm{LOS}}$, following standard practice due to the existing symmetry along the line of sight (e.g. Mahajan et al. 2011; Rhee et al. 2017; Pasquali et al. 2019; Rhee et al. 2020). The radial distance is given in units of $\mathrm{R}_{200}$ and the velocity axis is normalized by the velocity dispersion of the cluster along the line-of-sight (simply $\sigma$, hereafter) in order to enable comparisons between different systems. The normalization is based on a homology hypothesis, that states that systems are structurally equal, aside from a characteristic radius and velocity dispersion.

A major concern in the PPS construction is that clusters in our sample have a large range of richness, ranging from 25 to 900 galaxies. To avoid biases due to the different number of members, we stack all the galaxies belonging to the same cluster class and luminosity regime into a single PPS. Hence, we end up with four stacked PPSs: two for G clusters (B and F) and two for NG clusters (B and F).

\subsection{Discretizing the Projected Phase Space}

The PPS approach enables the identification of cluster members according to their infall time. Although in the full 6D phase space galaxies have well-defined trajectories as they enter the respective cluster (e.g. Wojtak \& Łokas 2010), in the PPS this trajectory is degenerate due to projection effects. Direct tracing of galaxies at different times since infall is not possible with observational data. Instead, cosmological simulations are used to define different regions in the PPS that correspond to the loci of galaxies at different times since infall. We make use of two different ways of slicing the PPS: 1) following R17, that use the YZiCS simulation to study the numeric density of galaxies at different times since infall occupying specific regions in the PPS. We refer to these regions as "Rhee Regions" hereafter. They provide a probabilistic approach to each region in the PPS; and 2) the separation presented in P19, also based on the YZiCS simulation, but defining analytic quadratic functions to fit the observed distributions of time since infall in the simulated stacked PPS. The PPS is then segmented in regions constraining galaxies to a narrow range of infall time, as done by P19, where the mean and the variance of each slice are listed in their Table 1. These PNZs (Pasquali New Zones, hereafter) are defined in decreasing mean infall time from PNZ 1 (innermost region, $\mathrm{t}_{\text {inf }} \sim 5.4 \mathrm{Gyr}$ ) to
PNZ $8\left(\mathrm{t}_{\text {inf }} \sim 1.4 \mathrm{Gyr}\right)$. Variances in the mean infall time of each PNZ range from $~ 1.5-2.5$ Gyr.

Although R17 and P19 use the same cosmological simulation (YZiCS), the way they slice the PPS is markedly different. The main differences are: 1) while R17 slice the PPS in 5 regions, P19 do it in 8 , 2) P19 do no include interlopers into their definition, while Rhee Regions take them into account (see Fig. 6 in R17); and 3) $\mathrm{P} 19$ limit their analysis to $\mathrm{R}_{200}$, while $\mathrm{R} 17$ extend to $2 \mathrm{R}_{200}$. We then compare how our results depend on the way we slice the PPS. We find the same trends between G and NG either using P19 or R17 approaches. Appendix A presents a more detailed discussion regarding this comparison. In this work, we use the P19 method of slicing the PPS due to its direct relation with infall time.

A word of caution is needed regarding the way the PPS is discretized, as in R17 and P19. We may ask which role is played by backsplash galaxies when we examine the PPS since they may suffer a partial quenching and have their stellar population properties modified when confronted to other galaxies in the cluster. Even using cosmological simulations it is not easy to define where these systems dominate in the PPS. For instance, Mahajan et al. (2011) find that the locus of their dominance is around $1.0 \leq \mathrm{R}_{\mathrm{LOS}} / \mathrm{R}_{200} \leq 1.5$ $\times 0 \leq\left|\mathrm{V}_{\mathrm{LOS}}\right| / \sigma \leq 1.0$, despite they represent only $\sim 30 \%$ of the galaxies in this box. To address the question of how backsplash galaxies can affect the results here obtained, we compare the distributions of backsplash galaxy properties in $\mathrm{G}$ and NG clusters similarly to Fig. 2. An important caveat is that in this case the distributions contain roughly $\sim 10$ times less points (galaxies) than the distributions shown in Figs. 2 and 3. Nevertheless, we find that backsplash galaxy properties have similar distributions compared to those shown above, especially in the faint regime. The similarity indicates that backsplash galaxies are affected by the environment just as any other set of galaxies. Therefore, comparison between galaxies within $R_{200}$ and the backsplash-limited ones (outside $R_{200}$ ) suggest that they do not influence our results significantly.

\subsection{Galaxy Distribution in the PPS}

In a first approximation, differences in cluster galaxy population may translate to a different distribution in the PPS. In this section, we explore how galaxies are distributed in the PPS of G and NG clusters. Table 2 shows the number of galaxies in each PNZ for $\mathrm{G}$ and NG systems, for both B and F regimes. Column (1) lists the cluster class; column (2) lists the luminosity regime; columns (3) to (10) show the number of galaxies in PNZs 1 to 8 , respectively; and column (11) lists how many galaxies are located beyond $R_{200}$. $41 \%$ of the $B$ galaxies in NG clusters are beyond $R_{200}$, while in $G$ clusters this percentage decreases to $28 \%$. F galaxies also show a similar trend: $28 \%$ and $34 \%$ of the $\mathrm{F}$ galaxies are beyond the $\mathrm{R}_{200}$ of $\mathrm{G}$ and NG clusters, respectively. These numbers unequivocally confirm that NG clusters show an excess of galaxies beyond $R_{200}$.

In Fig. 4 we show the normalized density of $B$ and $F$ galaxies in the PPS of G and NG clusters. We limit the PPS to $R_{200}$, where the PNZs are defined and enable a connection between infall time and PPS location. We divide the PPS into bins of $0.15\left|\mathrm{~V}_{\mathrm{LOS}}\right| / \sigma \times$ $0.05 R_{\mathrm{LOS}} / \mathrm{R}_{200}$ to guarantee a good sampling of the PNZs, adapting the bins to the aspect ratio of the diagram (note the ordinate extends a factor of 3 with respect to the abscissa, in normalized units). This slicing results in 12 and $6 \mathrm{~B}$ galaxies per bin (on average) in $\mathrm{G}$ and NG clusters, respectively. In the faint regime, we find lower values ( $\sim 5$ galaxies per bin in both cases). We use these bins to map the distribution of galaxies in PPS, performing a convolution in the resulting matrix (defined by the number of galaxies per bin) 

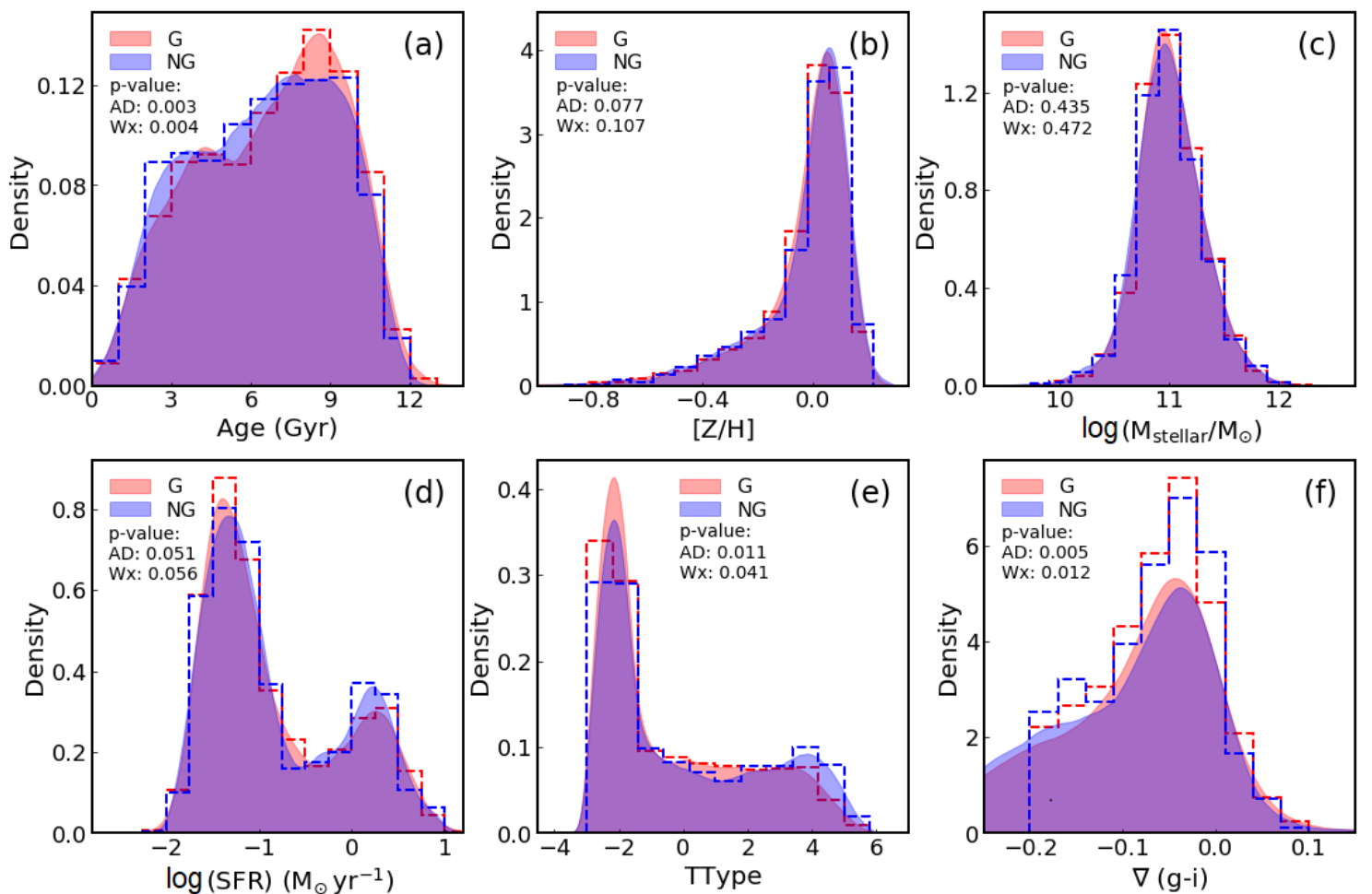

Figure 2. Distributions of age (a), $[\mathrm{Z} / \mathrm{H}](\mathrm{b}), \log \left(\mathrm{M}_{\mathrm{stellar}}\right)(\mathrm{c}), \log (\mathrm{SFR})(\mathrm{d}), \mathrm{TType}(\mathrm{e})$ and $\nabla(\mathrm{g}-\mathrm{i})$ (f) for bright galaxies in $\mathrm{G}$ and NG clusters. In each panel we also show the resulting p-values of an $\mathrm{AD}$ and Wx statistical test. The distributions of Age and TType of BG are statistically different between $\mathrm{G}$ and NG clusters.
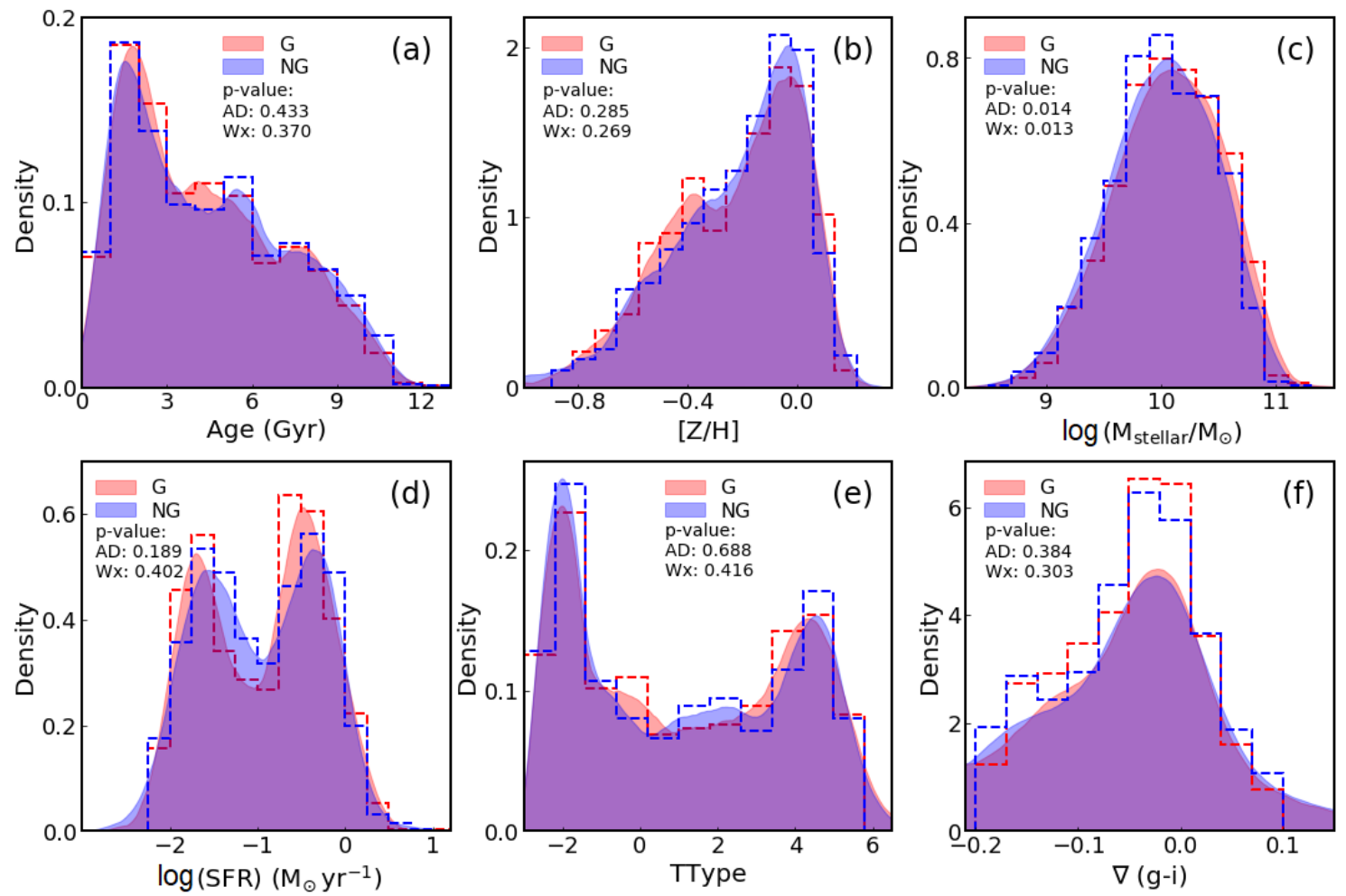

Figure 3. The same as Figure 2, but for F galaxies. Differently from the bright regime case, only $\log \left(\mathrm{M}_{\text {stellar }}\right)$ have statistically different distributions. 
Table 2. Number of galaxies in each PNZ.

\begin{tabular}{c|c|ccccccccc}
\hline & PNZ & 1 & 2 & 3 & 4 & 5 & 6 & 7 & 8 & $\mathrm{R}>\mathrm{R}_{200}$ \\
\hline \multirow{2}{*}{$\mathrm{G}$} & BG & 392 & 611 & 723 & 824 & 391 & 176 & 93 & 255 & 1352 \\
& FG & 58 & 125 & 249 & 150 & 66 & 33 & 19 & 41 & 289 \\
\hline \multirow{2}{*}{ NG } & BG & 101 & 157 & 126 & 229 & 141 & 66 & 26 & 69 & 623 \\
& FG & 47 & 128 & 182 & 249 & 119 & 52 & 22 & 58 & 441 \\
\hline
\end{tabular}

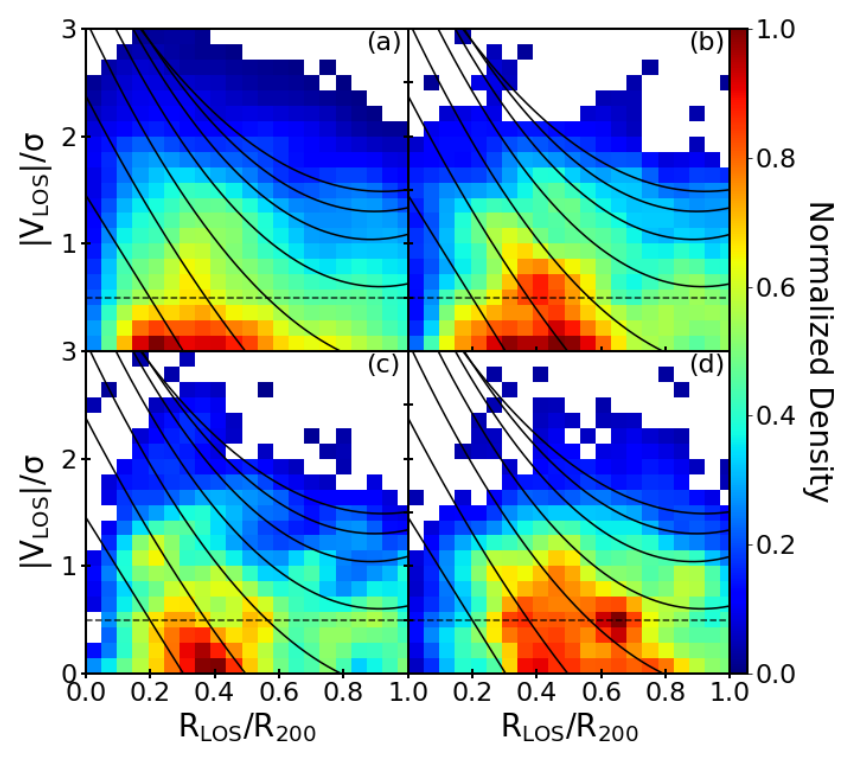

Figure 4. Normalized density distribution of galaxies in the PPS of G clusters (left) and NG clusters (right). We separate galaxies according to the two adopted luminosity regimes: bright on top panels and faint in the bottom panels. We note that for NG clusters, higher density regions extend to higher velocities in comparison to $\mathrm{G}$ clusters. We highlight this trend by including a $\left|\mathrm{V}_{\operatorname{LOS}}\right| / \sigma=0.5$ dashed line in the four panels.

using a Gaussian kernel with FWHM $=1.88\left(\sigma_{\text {std }}=1\right)^{6}$. The kernel size is defined to slightly reduce the noise and preserve the global trends. Bins without galaxies are carefully treated, applying an interpolation in "empty" bins that have at least 50\% of their neighbouring bins occupied. Note that by applying this interpolation scheme, we assume that transitions within these bins in PPS space are sufficiently smooth.

We note in Fig. 4 that indistinctly for $\mathrm{B}$ or $\mathrm{F}$ galaxies and $\mathrm{G}$ or NG clusters there is an offset of approximately $\mathrm{R}_{\mathrm{LOS}} \sim 0.4 \mathrm{R}_{200}$ between the cluster center and the higher density envelope (redder colors). This offset may be due to projection effects or a limitation when defining the cluster centers with galaxies, that represent a minor fraction of the cluster mass, compared to the gas and dark matter components. This issue lies beyond the scope of this paper but will be further investigated in future work (Sampaio et al. in prep). Despite this offset, the galaxy distributions are in agreement with both observational and simulated data from Rhee et al. (2020), guaranteeing that this offset does not represent a sample bias.

Comparison between galaxy distributions of $\mathrm{G}$ and NG clusters in the PPS shows that the higher density envelope (redder colors) in NG clusters extends to higher velocities than in the $\mathrm{G}$ case, for both

6 The FWHM/standard deviation was empirically defined, but does not affect the resulting trends.
$\mathrm{B}$ and $\mathrm{F}$ (see the dashed line in Fig. 4). We give special attention to PNZ 8, where we expect to find first infalling galaxies. However, it is important to consider that, in both B and F regimes, $\mathrm{G}$ and NG clusters have a different number of galaxies (see Section 2.2). To detail differences in PNZ 8 we then follow: 1) from the cluster class with more galaxies ( $\mathrm{G}$ in $\mathrm{B}$ and $\mathrm{NG}$ in $\mathrm{F}$ ) we randomly select $\mathrm{N}$ galaxies, where $\mathrm{N}$ is the number of galaxies in the less rich class (NG in B and G in F); 2) calculate the ratio of galaxies in the PNZ 8 between the equal-size samples; and 3) we repeat this procedure 1,000 times. This guarantees that differences of $\mathrm{G}$ and NG cluster PNZ 8 are not due to one sample being larger. A comparison shows that $\mathrm{G}$ clusters have an excess of $\sim 27 \%$ of B galaxies in the PNZ 8 with respect to NG clusters. In the faint regime, we find that PNZ 8 is roughly equally occupied in both cases. Namely, we find a mean ratio of 0.99 .

Regarding the excess of bright galaxies in the PNZ 8 of $\mathrm{G}$ systems with respect to NG ones, no firm conclusion can be drawn. However, we should stress that $\mathrm{dC} 17$ (see their Figure 7) find a higher kurtosis for the bright galaxies in G systems with respect to the NG ones. Datasets with high kurtosis tend to have heavy tails while datasets with low kurtosis tend to have light tails. Although this is not an explanation, it shows consistency. Furthermore, normally we would expect a more populated "virialized core", i.e. low PNZ number in $\mathrm{G}$ clusters. What we find is that $\mathrm{NG}$ clusters have fewer galaxies in high PNZ regions, i.e. mainly at high $\mathrm{V}_{\mathrm{LOS}} / \sigma$, so this would mean that the distributions of NG clusters are sub-gaussian, or platykurtic. The reason as to why the kurtosis is negative needs further investigation. The point above justifies that NG systems have higher $\sigma$ (as an equivalent width of the velocity distribution), but not necessarily that the tails of the distribution are filled with respect to Gaussians, i.e. not enough high-velocity galaxies as they are still in a preliminary infall state. We plan on investigating this particular issue using cosmological simulations like Illustris and YZiCS in a future work.

\section{EXPLORING VARIOUS GALAXY PROPERTIES IN PROJECTED PHASE SPACE}

Environmental quenching drastically affects galaxy evolution. Previous works show that the quenched fraction of galaxies within clusters is a function of clustercentric radius (e.g., Sazonova et al. 2020). However, RPS and TML (see Section 1) are also conditional on the incoming velocity of the infalling galaxy (see R19 for an example). The PPS provides a powerful tool to understand how galaxy properties are affected in high-density environments, taking into account both velocity and position. In this section, we study the distribution on the PPS of median age, $[\mathrm{Z} / \mathrm{H}]$, SFR, TType, $\nabla(\mathrm{g}-\mathrm{i})$, $\mathrm{M}_{\text {stellar }}$ in $\mathrm{G}$ and NG clusters. We use a similar approach as in Fig. 4 to study the corresponding distributions. Furthermore, we calculate the variance in each pixel for each parameter to compare with measurement errors. In general, the variance in the pixel is greater than the measurement errors. For instance, we find that the map of the B galaxies Age has a mean variance of $0.74 \mathrm{Gyr}$. In the following, we describe differences greater than the mean variance.

Fig. 5 displays (from top to bottom) the distribution on the PPS of median stellar age, $[\mathrm{Z} / \mathrm{H}], \log (\mathrm{SFR})$, TType, and $\nabla(\mathrm{g}-\mathrm{i})$ for $\mathrm{B}$ (leftmost two columns) and $\mathrm{F}$ (rightmost two columns) galaxies, in $\mathrm{G}$ and NG clusters, as labeled. The regions delimiting the PNZs are shown as solid lines and classify galaxies according to their infall times. We tested our results against statistical noise in the following way: 1) we randomly divide our sample (177) into two 
halves; 2) we perform the same analysis as in Fig. 5; and 3) compare the differences between the two half-samples. When clusters are randomly chosen we do not find significant differences. This ensures that the differences between $\mathrm{G}$ and NG clusters are not due to statistical noise. We also use this test as guidance to define the significant differences between $\mathrm{G}$ and NG clusters.

Comparison of B galaxy property distributions in $\mathrm{G}$ and $\mathrm{NG}$ clusters (two first columns of Fig. 5) shows some general trends. In summary, we find: 1) NG clusters have more mixed distributions, whereas $\mathrm{G}$ clusters have smoother distributions concerning the PNZs; 2) we find that both $[\mathrm{Z} / \mathrm{H}]$ and color gradient show an almost uniform distribution over the PPS, indistinctly for $\mathrm{G}$ and NG clusters; and 3) B galaxies with high $\left|\mathrm{V}_{\mathrm{LOS}}\right| / \sigma$ in PNZs $<4$ of $\mathrm{G}$ clusters (see Section 4.2) are older, less star-forming and more elliptical than its counterpart in NG cluster. More quantitatively, a comparison of panels (a) and (b) shows that the (inner) PNZs $\leq 4$ of NG cluster have galaxies with age $<7 \mathrm{Gyr}$, while the equivalent in $\mathrm{G}$ clusters is dominated by older galaxies, with age $>7$ Gyr. Namely, $70 \%$ of the $G_{B}$ galaxies in PNZ $\leq 4$ regions have age $>7 \mathrm{Gyr}$, whereas for $\mathrm{NG}_{\mathrm{B}}$ clusters this percentage is $53 \%$. In panels (e) and (f), the mean $[\mathrm{Z} / \mathrm{H}]$ of $\mathrm{G}_{\mathrm{B}}$ and $\mathrm{NG}_{\mathrm{B}}$ galaxies is $-0.020 \pm 0.001^{7}$ and $-0.010 \pm 0.002$, respectively. Regarding SFR, both $\mathrm{G}$ and NG clusters show a trend of lower SFR with higher infall time (i.e. decreasing PNZ). The quenching is defined by a decrease in the SFR and this result highlights the cumulative effect of environmental quenching with respect to the time since infall. Despite this overall behaviour, a comparison of panels (i) and (j) shows that, $\mathrm{G}_{\mathrm{B}}$ galaxies (panel i) with $\log (\mathrm{SFR})<-0.6$ occupy mainly PNZs 1 to 4 . In contrast, the equivalent cut in $N_{B}$ galaxies (panel $\mathrm{j}$ ) restricts the PPS to PNZs 1 to 3 . In the PNZ 4 of NG clusters, we note an excess of galaxies with higher SFR in comparison to their counterparts in $\mathrm{G}$ systems. Examining the B galaxies morphological, TType distribution (panels $\mathrm{m}$ and $\mathrm{n}$ ) we find similarities with the distributions of SFR, $[\mathrm{Z} / \mathrm{H}]$ and Age, especially in the $\mathrm{G}$ case. This provides further evidence that the quenching of star formation correlates with morphological transformation. TType differences between $\mathrm{G}$ and $\mathrm{NG}$ clusters are restricted mainly to PNZs $\leq 4$. We find that $\mathrm{G}_{\mathrm{B}}$ galaxies in the $\mathrm{PNZ} \leq 4$ region have an average TType value of $-1.2 \pm 0.2$, substantially lower than the equivalent average in $\mathrm{NG}_{\mathrm{B}}$ galaxies $(-0.5 \pm 0.2)$. In the $\mathrm{PNZ}>4$ areas, the mean difference between $\mathrm{G}$ and $\mathrm{NG}$ decreases to $\Delta\langle$ TType $\rangle(\mathrm{G}-\mathrm{NG}) \sim 0.2$ ( $\left\langle\right.$ TType $\left._{\mathrm{PNZ}>4}\right\rangle=-0.1$ and 0.1 for $\mathrm{G}$ and $\mathrm{NG}$ clusters, respectively). Finally, the distribution of color gradient is similar in $\mathrm{G}$ and NG clusters (panels q and f). Galaxies with $\nabla(\mathrm{g}-\mathrm{i})<-0.1$ are found mainly in PNZ 8 and at $\left|\mathrm{V}_{\mathrm{LOS}}\right| / \sigma>1.7$. Lower values of color gradient mean bluer outskirts (see eq. 3). This trend suggests that $\mathrm{B}$ galaxies enter clusters with bluer colors on the outside and the difference decreases after traversing the PPS to PNZ 6.

The PPS analysis shows that, further to the differences found in the sample of B galaxies, we note more striking ones in the faint regime (two last columns of Fig. 5). We highlight the main differences between $\mathrm{F}$ galaxies in $\mathrm{G}$ and $\mathrm{NG}$ clusters with red ellipses in Fig. 5. Our results suggest that $F$ galaxies (hence less massive) are mostly quenched by environmental effects, in agreement with works showing the stellar mass dependence on galaxy evolution (Peng et al. 2010; Wetzel et al. 2012b). In panels (c) and (d), we note that

\footnotetext{
7 Hereafter the shown values are calculated using the galaxy distribution itself, instead of the pixellated version of the PPS. However, comparison of the two methods shows differences lower than $5 \%$ in both mean value and error.
}

$\mathrm{NG}_{\mathrm{F}}$ galaxies in the highlighted region are on average $1.4 \pm 0.4 \mathrm{Gyr}$ younger than in the $\mathrm{G}_{\mathrm{F}}$ case. On the other hand, $\mathrm{NG}_{\mathrm{F}}$ galaxies beyond $0.6 R_{200}$ are slightly older by, on average, $0.9 \pm 0.2 \mathrm{Gyr}$ concerning to $\mathrm{G}_{\mathrm{F}}$ galaxies. Panels $(\mathrm{g})$ and $(\mathrm{h})$ show significant differences in average metallicity between $G$ and NG clusters. $G_{F}$ galaxies in the red highlighted region are more-metal-poor by, on average, $0.28 \pm 0.04$ dex with respect to $\mathrm{NG}_{\mathrm{F}}$ galaxies. However, within $0.6 R_{200}$ there are no significant differences between $G$ and $\mathrm{NG}$ clusters. More quantitatively, we find that $\mathrm{G}_{\mathrm{F}}$ galaxies within $0.6 R_{200}$ are on average $0.010 \pm 0.002$ dex more metal-rich than the $\mathrm{NG}_{\mathrm{F}}$ counterparts, comparable to the uncertainty shown in Table 1 . These results are in agreement with $\mathrm{dC} 17$, which further show that differences in the outskirt extend to $2 \mathrm{R}_{200}$. Regarding $\mathrm{F}$ galaxies $\mathrm{SFR}$, a trend of a core with quenched galaxies with a tail to higher velocities is visible in panels $(\mathrm{k})$ and (l). In panel $(\mathrm{k})$, we note a tail in the distribution with $\log (\mathrm{SFR})<-1.0$ that extends up to $\sim 1.7\left|\mathrm{~V}_{\mathrm{LOS}}\right| / \sigma$. In NG clusters (panel $\mathrm{k}$ ) this tail is more conspicuous. NG clusters also appear more mixed. Namely, we note a trail of quenched galaxies in $0.5 \mathrm{R}_{200}<\mathrm{R}_{\mathrm{LOS}}<0.7 \mathrm{R}_{200}$ that extends to $\sim 1.7\left|\mathrm{~V}_{\mathrm{LOS}}\right| / \sigma$ and that only PNZ 1 is fully occupied by galaxies with $\log (\mathrm{SFR})<-1.2$. As in the B regime, we note relevant similarities between the SFR, $[\mathrm{Z} / \mathrm{H}]$, Age and TType distributions in both $\mathrm{G}$ and NG clusters, which further support the relation between morphology and stellar population properties. Regarding $\mathrm{G}$ vs NG, panels (o) and (p) show that $\mathrm{G}_{\mathrm{F}}$ within the red ellipse in $\mathrm{G}$ clusters have higher TTypes compared to the same subset in NG clusters. Namely, we find an average TType value of $2.0 \pm 0.4$ and $0.9 \pm 0.4$ for $\mathrm{G}$ and NG clusters, respectively. At last, examining panels (s) and $(\mathrm{t})$ we find that $\mathrm{NG}$ clusters have an excess of $\mathrm{F}$ galaxies with $\nabla(\mathrm{g}-\mathrm{i})>0$. This subset of galaxies (highlighted by the red ellipse) is found in every PNZ, which may indicate that these F galaxies entered the NG cluster already with shallow/positive values of color gradients.

In Fig. 6 we present the median $M_{\text {stellar }}$ distribution in the PPS of G and NG clusters, with galaxies divided into B and F. We separate this specific parameter from the last 5 discussed since in this case the B and F split translates, by construction, into different ranges in stellar mass. Therefore, we select suitable ranges for each luminosity regime, while guaranteeing that both have the same relative difference in $\log \left(\mathrm{M}_{\text {stellar }} / \mathrm{M}_{\odot}\right)$. The plotting ranges are defined from the distribution of $\log \left(\mathrm{M}_{\text {stellar }} / \mathrm{M}_{\odot}\right)$, as shown in Figs. 2 and 3. Comparing panels (a) and (b), we see that the distribution of $\log \left(\mathrm{M}_{\text {stellar }} / \mathrm{M}_{\odot}\right)$ in $\mathrm{G}$ and $\mathrm{NG}$ clusters for $\mathrm{B}$ galaxies are quite similar. We observe that in both $\mathrm{G}$ and $\mathrm{NG}$ clusters, only $\sim 34.5 \%$ of $\mathrm{B}$ galaxies have $\log \left(\mathrm{M}_{\text {stellar }} / \mathrm{M}_{\odot}\right)>11.1$. In the faint regime, we note that approximately $48 \%$ of the $\mathrm{F}$ galaxies have $\log \left(\mathrm{M}_{\text {stellar }} / \mathrm{M}_{\odot}\right)>10.1$, corresponding to the upper part of the defined range. In Section 8, we discuss this difference between B and $\mathrm{F}$ galaxies in the context of the downsizing model (Neistein et al. 2006). In the faint regime, we also note significant differences between $\mathrm{G}$ and NG clusters. Panel (c) shows that within $0.3 \mathrm{R}_{200}$ there is an excess of $\mathrm{F}$ galaxies with $\log \left(\mathrm{M}_{\text {stellar }} / \mathrm{M}_{\odot}\right)>10.2$ in $\mathrm{G}$ clusters. Additionally, in panel (d) we note within $0.4 \mathrm{R}_{200} \mathrm{NG}$ clusters feature an excess of $\mathrm{F}$ galaxies with $\log \left(\mathrm{M}_{\text {stellar }} / \mathrm{M}_{\odot}\right)<10.0$.

We find that galaxy property distributions in $\mathrm{G}$ and NG clusters are significantly different from the PPS point of view. Despite global similarities (see Section 3), the PPS analysis shows they are differently distributed in such systems. Furthermore, we find good agreement between galaxy properties variation in the PPS and PNZs (and infall time, consequently). This suggests a more straightforward relation between galaxy properties and infall time as a consequence of the cumulative effect of environmental quenching. 


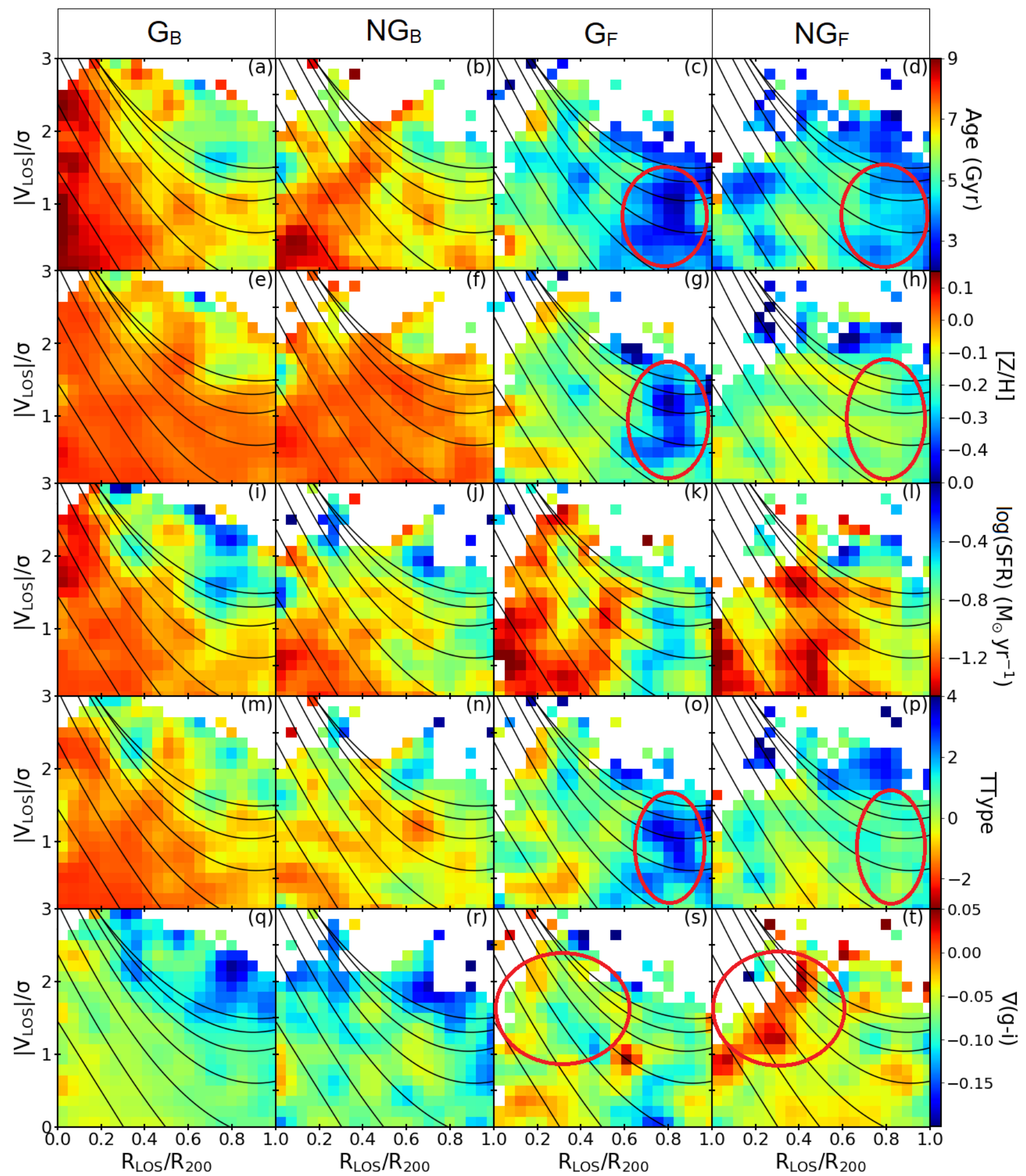

Figure 5. From top to bottom we present the distribution of age, $[\mathrm{Z} / \mathrm{H}], \log (\mathrm{SFR})$, TType and $\nabla(\mathrm{g}-\mathrm{i})$, respectively, over the PPS of G and NG clusters and luminosity regime (B or F). The red ellipses highlight differences between galaxy properties in $\mathrm{G}$ and $\mathrm{NG}$ clusters, in the faint regime. 


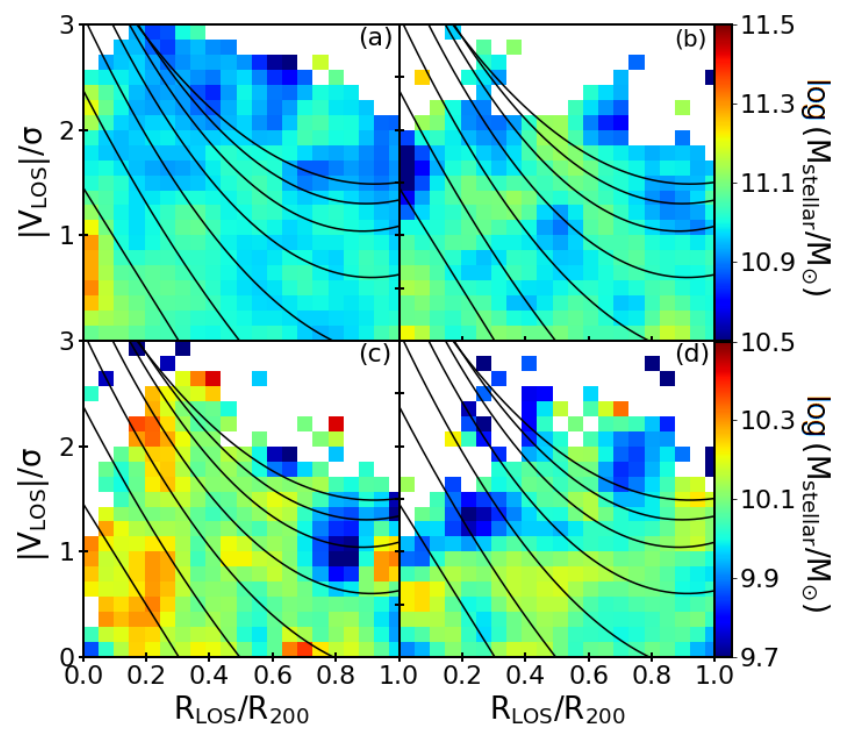

Figure 6. Panels (a) and (b): B galaxies $M_{\text {stellar }}$ distribution over the PPS of G and NG clusters, respectively. Panels (c) and (d): the same, but for F galaxies.

\section{THE RELATION BETWEEN GALAXY PROPERTIES AND INFALL TIME IN G AND NG CLUSTERS}

In this section, we explore in more detail the differences found in the distribution of $\mathrm{G}$ and NG clusters on the PPS diagram, presented in Section 5. The delayed-then-rapid model (Wetzel et al. 2013) proposes that galaxy quenching depends on $t_{\text {inf }}$. We probe environmental effects on galaxies by studying the relation between $\mathrm{t}_{\mathrm{inf}}$ and galaxy properties in $\mathrm{G}$ and NG systems, separating galaxy populations into $\mathrm{B}$ and $\mathrm{F}$. We consider that all galaxies in a single PNZ are well represented by the mean $t_{\text {inf }}$ presented in P19. We show in Fig. 7, in panels (a) to (f), the median values of age, [Z/H], $\log \left(\mathrm{M}_{\text {stellar }} / \mathrm{M}_{\odot}\right), \log (\mathrm{SFR})$, TType and $\nabla(\mathrm{g}-\mathrm{i})$, respectively, for galaxies in each PNZ. In order to highlight global trends, we perform a spline fitting, which better shows the relation between infall time and galaxy properties. The curve width in Fig. 7 represents the associated 1- $\sigma$ error in each PNZ. To address the statistical variance we use a bootstrap technique. We follow: 1) for each PNZ we randomly select $\mathrm{N}$ values (where $\mathrm{N}$ is the number of galaxies in the same region), with replacement, from the observed distribution; 2) calculate the variance using the new distribution $Q_{\text {sigma }}{ }^{8} ; 3$ ) we repeat this procedure 1,000 times; and 4) consider the variance as the median of the $\mathrm{Q}_{\text {sigma }}$ distribution. Additionally, we perform a similar analysis to the mass matched sample in the bright regime, in order to investigate how the mass mismatch between $\mathrm{G}$ and NG clusters (see Section 3) affects our results. Quantitatively, we find mean differences ${ }^{9}$ of $0.06 \mathrm{Gyr}, 0.001 \mathrm{dex}, 0.01 \mathrm{dex}, 0.02 \mathrm{dex}, 0.05$ and 0.03 for Age, $[\mathrm{Z} / \mathrm{H}], \log \left(\mathrm{M}_{\text {stellar }} / \mathrm{M}_{\odot}\right), \log (\mathrm{SFR})$, TType and $\nabla(\mathrm{g}-\mathrm{i})$, respectively. Comparison with the uncertainties shown in Table 1 guarantees that the results shown in Fig. 8 do not depend on the use of mass-matched samples.

In a first approximation, we adopt a linear relation between $t_{\text {inf }}$ and galaxy properties. However, we emphasize that this is a

${ }^{8}$ The variance from quartiles is calculated as $\mathrm{Q}_{\text {sigma }}=0.74 \times\left(\mathrm{Q}_{75 \%}-\right.$ $\mathrm{Q}_{25 \%}$ ).

9 Over all PNZs for G and NG clusters. functional approach and the relations are not expected to be linear. Our goal here is to derive quantities such as variations with infall time. We fit the observed relations via the ScIDAV Is statistical analysis tool (Benkert et al. 2014), that takes into account errors in both $\mathrm{x}$ and $\mathrm{y}$ for the fit. The resulting intercept and gradient coefficients are shown in Table 3. Column (1) lists cluster class and luminosity regime; in column (2) we list the correspondent coefficient; columns (3) to (8) show the results and associated errors for age, $[\mathrm{Z} / \mathrm{H}], \log \left(\mathrm{M}_{\text {stellar }} / \mathrm{M}_{\odot}\right), \log (\mathrm{SFR})$, TType and $\nabla(\mathrm{g}-\mathrm{i})$, respectively. We compare the significance level of the differences between $\mathrm{G}$ and $\mathrm{NG}$ clusters as:

$\sigma=\frac{\sqrt{\left(\sigma_{\mathrm{i}, \mathrm{G}}^{\chi}\right)^{2}+\left(\sigma_{\mathrm{i}, \mathrm{NG}}^{\chi}\right)^{2}}}{2}$,

where $\sigma_{\mathrm{i}, \mathrm{G}}^{\chi}$ and $\sigma_{\mathrm{i}, \mathrm{NG}}^{\chi}$ are the errors associated with the i-th coefficient (linear or angular) of the parameter $\chi$ (age, for example). In Table 3, we highlight in red relevant differences in the gradients of $\mathrm{G}$ and NG clusters. Dark red means differences greater than 2- $\sigma$ and light red differences greater than 1- $\sigma$. We highlight similarly differences in the intercept coefficients in blue color.

With respect to global trends, we see from Fig. 7 that the median stellar population parameters (panels $a, b$ and c) differ significantly between B and F galaxies, both in $\mathrm{G}$ and NG systems. For instance, we find a mean difference of $2.2 \pm 0.5 \mathrm{Gyr}, 0.3 \pm 0.1$ and $0.99 \pm 0.06$ dex between the intercept parameter of $\mathrm{B}$ and $\mathrm{F}$ galaxies for age, $[\mathrm{Z} / \mathrm{H}]$ and $\log \left(\mathrm{M}_{\text {stellar }} / \mathrm{M}_{\odot}\right)$, respectively. Additionally, the relation with respect to SFR of $\mathrm{B}$ galaxies has a gradient closer to zero than the one for $\mathrm{F}$ galaxies. We find an average slope ${ }^{10}$ of -0.08 and -0.20 for the SFR of B and F galaxies, respectively. These results unequivocally suggest that $\mathrm{B}$ and $\mathrm{F}$ galaxies are distinctly affected by environmental quenching. However, we find that indistinctly in $\mathrm{G}$ and NG clusters, both B and F galaxies feature similar trends in stellar mass (within 0.1 dex, comparable with the measurement uncertainty), and the trend is approximately constant with infall time. This result may suggest that galaxy quenching is mostly related to the removal of the gas component instead of the stellar mass itself.

In panel (a) of Fig. 7, two noticeable trends can be found regarding the evolution of galaxy properties with $t_{\text {inf }}$ in $G$ and NG clusters. First, $G_{B}$ galaxies with $t_{\text {inf }} \geq 3.80 \mathrm{Gyr}$ are on average $0.3 \pm 0.2$ Gyr older than $\mathrm{NG}_{\mathrm{B}}$ systems. Furthermore, we find that at $\mathrm{t}_{\mathrm{inf}}<4.8 \mathrm{Gyr} \mathrm{NG}$ Falaxies are on average $0.6 \pm 0.5 \mathrm{Gyr}$ older than those in $\mathrm{G}_{\mathrm{F}}$. The third column of Table 3 shows that in both bright and faint regimes, the slope of the relation between age and $t_{\text {inf }}$ in NG clusters lies more than 1- $\sigma$ lower than that for G clusters. In panel (b) we see that $[\mathrm{Z} / \mathrm{H}]$ behaves similarly. Despite no significant difference for B galaxies, $F$ galaxies with $t_{\text {inf }}<4.8 \mathrm{Gyr}$ in NG clusters are $0.05 \pm 0.02$ dex more metal-rich than in $G$ clusters. We also note a difference greater than $2-\sigma$ in the slope of the $[\mathrm{Z} / \mathrm{H}]$ relation in G and NG clusters. Namely, we find a shallower relation in NG clusters compared to G systems (see Table 3, fourth column). In other words, the trends found in panels (a) and (b) suggest that F galaxies with $4.5<\mathrm{t}_{\text {inf }}<1.5 \mathrm{Gyr}$ in NG clusters are older and more metal-rich than in $\mathrm{G}$ clusters. On the other hand, the excess of $B$ galaxies that have younger ages at high infall time in NG clusters may suggest that $\mathrm{G}$ clusters have a better defined virialized core, as we will discuss in Section 8. In panel (c), we do see that $\mathrm{M}_{\text {stellar }}$ is approximately constant with infall time, indistinctly of cluster class and luminosity regime. This translates to slopes lower than $0.1 \mathrm{dex}$ in all cases. In panel (d), the SFR is shown as a function of infall

10 Between G and NG clusters. 

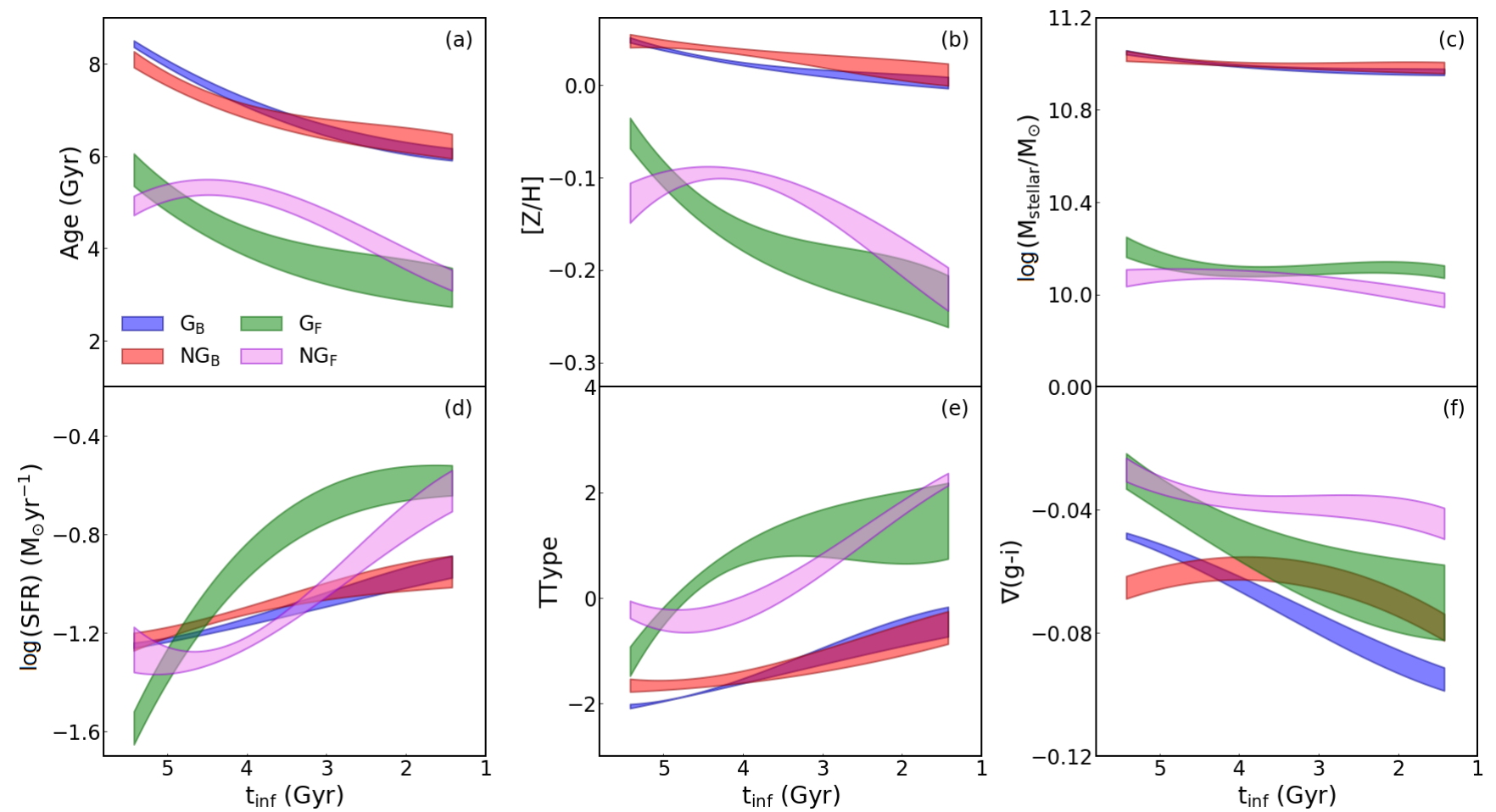

Figure 7. From panel (a) to (f): Relation between time since infall and age, $[\mathrm{Z} / \mathrm{H}], \log \left(\mathrm{M}_{\text {stellar }} / \mathrm{M}_{\text {odot }}\right), \log (\mathrm{SFR})$, TType and $\nabla(\mathrm{g}-\mathrm{i})$ of galaxy members of $\mathrm{G}$ and NG clusters. Galaxy members are split into bright (red/blue) or faint (green/pink). The curves and its width represent a cubic spline fitting to the median values and the variance calculated using the bootstrap technique, respectively.

Table 3. Gradient and Intercept Coefficients from the linear fit presented in Section 6.

\begin{tabular}{c|c|cccccc}
\hline & $\mathrm{y}=\mathrm{ax}+\mathrm{b}$ & Age $(\mathrm{Gyr})$ & {$[\mathrm{Z} / \mathrm{H}]$} & $\log \left(\mathrm{M}_{\text {stellar }} / \mathrm{M}_{\odot}\right)$ & $\log (\mathrm{SFR})\left(\mathrm{M}_{\odot} / \mathrm{yr}\right)$ & TType & $\nabla(\mathrm{g}-\mathrm{i})$ \\
\hline \multirow{2}{*}{$\mathrm{G}_{\mathrm{B}}$} & $\mathrm{a}$ & $0.701 \pm 0.052$ & $0.014 \pm 0.002$ & $0.025 \pm 0.006$ & $-0.078 \pm 0.011$ & $-0.333 \pm 0.042$ & $0.012 \pm 0.001$ \\
---- & $\mathrm{b}$ & $4.468 \pm 0.219$ & $-0.031 \pm 0.008$ & $10.896 \pm 0.023$ & $-0.832 \pm 0.051$ & $-0.266 \pm 0.201$ & $-0.115 \pm 0.006$ \\
$\mathrm{NG}_{\mathrm{B}}$ & $\mathrm{a}$ & $0.538 \pm 0.105$ & $0.013 \pm 0.004$ & $0.019 \pm 0.010$ & $-0.100 \pm 0.020$ & $-0.455 \pm 0.168$ & $0.003 \pm 0.006$ \\
& $\mathrm{~b}$ & $4.826 \pm 0.448$ & $-0.022 \pm 0.014$ & $10.925 \pm 0.041$ & $-0.706 \pm 0.089$ & $0.553 \pm 0.643$ & $-0.078 \pm 0.009$ \\
\hline \multirow{2}{*}{$\mathrm{G}_{\mathrm{F}}$} & $\mathrm{a}$ & $0.626 \pm 0.068$ & $0.052 \pm 0.010$ & $0.037 \pm 0.012$ & $-0.249 \pm 0.027$ & $-0.611 \pm 0.151$ & $0.009 \pm 0.004$ \\
---- & $\mathrm{b}$ & $2.043 \pm 0.705$ & $-0.360 \pm 0.040$ & $9.973 \pm 0.043$ & $-0.139 \pm 0.110$ & $2.497 \pm 0.661$ & $-0.080 \pm 0.020$ \\
$\mathrm{NG}_{\mathrm{F}}$ & $\mathrm{a}$ & $0.511 \pm 0.101$ & $0.021 \pm 0.010$ & $0.035 \pm 0.012$ & $-0.141 \pm 0.038$ & $-0.755 \pm 0.079$ & $0.005 \pm 0.002$ \\
& $\mathrm{~b}$ & $2.888 \pm 0.446$ & $-0.192 \pm 0.039$ & $9.928 \pm 0.046$ & $-0.692 \pm 0.162$ & $3.257 \pm 0.275$ & $-0.057 \pm 0.010$ \\
\hline
\end{tabular}

time. SFR behaves similarly to $[\mathrm{Z} / \mathrm{H}]$. In the bright regime, we note that galaxies do not show significant differences in SFR in $G$ and NG clusters. However, the SFR slope for G and NG clusters differs by more than $1-\sigma$. These differences are more evident in the faint regime: at $\mathrm{t}_{\mathrm{inf}}<4.8 \mathrm{Gyr} \mathrm{NG}_{\mathrm{F}}$ galaxies are less star-forming by, on average, $0.21 \pm 0.17$ dex than $\mathrm{G}_{\mathrm{F}}$ systems. The slope of the SFR relation differs more than 2- $\sigma$ between G and NG clusters. Similarly to age and $[\mathrm{Z} / \mathrm{H}]$, NG clusters feature shallower slopes in comparison to $\mathrm{G}$ clusters, therefore implying a weak dependence with infall time. Panel (e) shows the trends with morphology (TType), appearing very similar to those for the SFR, supporting the relation between star formation quenching and morphological transition. $\mathrm{F}$ galaxies with $t_{\text {inf }}<4.8 \mathrm{Gyr}$ in NG clusters have lower values of TType, $0.27 \pm 0.09$ on average, in comparison to $\mathrm{G}$ clusters. Finally, panel (f) plots the relation between $\nabla(\mathrm{g}-\mathrm{i})$ and infall time.
Significant differences are found in both $\mathrm{B}$ and $\mathrm{F}$ subsets. In the bright regime, $\mathrm{G}_{\mathrm{B}}$ galaxies show a constantly increasing (negative) color gradient with infall time, while $\mathrm{NG}_{\mathrm{B}}$ galaxies show a plateau after $t_{\text {inf }} \sim 2.5 \mathrm{Gyr}$. The slopes of these two relations differ more than $1-\sigma$, as can be seen in Table 3. In the faint regime, we note that $\mathrm{NG}_{\mathrm{F}}$ galaxies have unequivocally shallower color gradients than $\mathrm{G}_{\mathrm{F}}$ systems. Quantitatively, we find a mean difference of 0.026 , being more negative in NG clusters.

\section{AN ESTIMATE OF THE INFALLING RATE IN NG CLUSTERS}

Several works relate the Non-Gaussianity of the velocity distribution with a higher infall rate in NG clusters compared to G systems (e.g. 
Roberts \& Parker 2017, de Carvalho et al. 2017). In this section, we present further evidence of a higher infall rate in NG systems by detailing the distribution of stellar mass in the PPS. Galaxies in the PNZ 8 (see Table 1 in P19) have an average infall time of $1.42 \mathrm{Gyr}$ and are mainly first infallers. However, the discretization of the PPS presented by P19 is limited to $\mathrm{R}_{200}$ and it is expected that galaxies first infalling in clusters may be also found beyond this threshold. Thus we decided to include the Rhee Region A in order to account for galaxies beyond $\mathrm{R}_{200}$ (see Fig. 6 of R17). This region is mostly occupied by interlopers. However, it is expected that the Shiftgapper technique returns a catalog of bona fide members. Hence, we consider that galaxies in the Rhee Region A correspond to the second most probable population, namely first infallers.

Differently from the previous analysis, here we consider the PPS for each cluster separately. We calculate the sum of stellar mass in PNZ 8 (or Rhee Region A) for each cluster and then take an average value for a given cluster class ( $\mathrm{G}$ or $\mathrm{NG}$ ) and luminosity regime (B or F). In the bright regime, NG clusters have an excess of stellar mass of $0.51 \times 10^{11} \mathrm{M}_{\odot}\left(\right.$ PNZ 8) and $0.84 \times 10^{12} \mathrm{M}_{\odot}$ (Rhee A), with respect to $\mathrm{G}$ clusters. In the faint regime, we also note NG clusters have an excess of $0.33 \times 10^{11} \mathrm{M}_{\odot}(\mathrm{PNZ} 8)$ and $0.31 \times 10^{12} \mathrm{M}_{\odot}$ (Rhee A) with respect to G clusters. Putting together the contributions of $\mathrm{B}$ and $\mathrm{F}$ galaxies, we find that $\mathrm{NG}$ clusters have an excess of $\sim 10^{11} \mathrm{M}_{\odot}$ in the PNZ 8 and $\sim 10^{12} \mathrm{M}_{\odot}$ in the Rhee Region A. This unambiguously shows that there are more galaxies infalling in NG clusters in comparison to $\mathrm{G}$ clusters.

Using the relation between locus in the PPS and infall time we can derive a rough estimate of the infall rate in NG clusters. We calculate the mean infall rate ( $\langle\mathrm{IR}\rangle)$ as follows: 1$)$ For each cluster we sum the stellar mass within a single PNZ and divide it by the mean infall range of PNZ i - PNZ i-1;2) we take the average value for each PNZ for a given cluster class and luminosity regime and 3) these estimates across all PNZ regions provide an infall history for $\mathrm{G}$ and NG clusters. They represent a rough estimate of the amount of stellar mass accreted into clusters from the infall time of PNZ i- 1 to the PNZ $\mathrm{i}$, with $\mathrm{i}$ varying from 1 to $8^{11}$ The results are shown in Fig. 8, where panels (a) and (b) correspond to B and F galaxies, respectively. We show at the bottom right of each panel the mean error for each case. We note that NG clusters feature larger stellar mass across all values of infall time. We find a mean difference of $\left\langle\langle\mathrm{IR}\rangle_{\mathrm{NG}}-\langle\mathrm{IR}\rangle_{\mathrm{G}}\right\rangle=$ $0.4 \times 10^{11} \mathrm{M}_{\odot}$ and $0.3 \times 10^{11} \mathrm{M}_{\odot}$ in the $\mathrm{B}$ and $\mathrm{F}$ regimes, respectively. An integration of the relation between $\langle\mathrm{IR}\rangle$ and $\mathrm{t}_{\text {inf }}$ suggests that NG clusters accreted $(1.5 \pm 0.8) \times 10^{12} \mathrm{M}_{\odot}$ more stellar mass in the last $\sim 5 \mathrm{Gyr}$ than $\mathrm{G}$ systems. For comparison, it roughly corresponds to the stellar mass of the local group, $\sim 10^{12} \mathrm{M}_{\odot}$.

A potential sample bias caveat relates to the different virial mass distributions of $\mathrm{G}$ and NG clusters, as shown in panel (a) of Fig. 1. In order to guarantee that NG clusters have a higher infall rate regardless of their mass, we separate our sample in fixed bins in $\log \left(\mathrm{M}_{200} / \mathrm{M}_{\odot}\right)$ from 14 to 14.75 in steps of 0.25 . The bin size is roughly half of the error $(\sim 0.13 \mathrm{dex})$ in $\log \left(\mathrm{M}_{200} / \mathrm{M}_{\odot}\right)$ (Yang et al. 2007, dC17). This guarantees that the mass distribution within each bin has no significant differences between $\mathrm{G}$ and $\mathrm{NG}$ clusters. The [14,14.75] range is chosen due to a limitation of the faint component of $G$ clusters. Namely, we do not find $G$ clusters in the $F$ regime with $\log \left(\mathrm{M}_{200} / \mathrm{M}_{\odot}>14.75\right)$. Thus this range guarantees that we have $\mathrm{G}$ and NG clusters in every bin for both luminosity regimes. The analysis reveals that, in all three bins, NG clusters have accreted more mass over the last $\sim 5 \mathrm{Gyr}$ in comparison to G clusters. Taking

11 At $\mathrm{i}=8$, the infall range is from 0 to $\mathrm{t}_{\text {inf }}(\mathrm{PNZ} 8)$
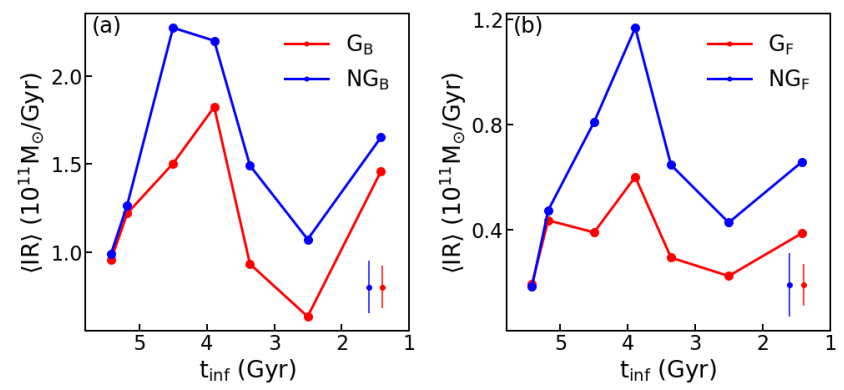

Figure 8. Estimate of mean infall rate of B (left) and F (right) luminosity regime as a function of $t_{\text {inf }}$ for G and NG clusters. Non-Gaussian systems show higher values of infall rate in both, with respect to $G$ systems.

the average over the three virial mass bins we find that NG clusters accreted an excess of $(1.4 \pm 0.7) \times 10^{11} \mathrm{M}_{\odot}$ and $(0.9 \pm 0.5) \times 10^{11} \mathrm{M}_{\odot}$ in the $\mathrm{B}$ and $\mathrm{F}$ regimes, respectively. These trends show that most of the difference originates in the tail end of the halo distribution, but confirms that the NG classification is directly related to a higher infall rate regardless of virial mass.

\section{DISCUSSION AND CONCLUSIONS}

\subsection{G and NG Clusters: Different Environments to Study Galaxy Evolution}

A comparison of $\mathrm{G}$ and $\mathrm{NG}$ clusters indicates that these two groups have significant differences in both structure and galaxy properties. We summarize those differences below:

- NG Clusters are more massive, larger (i.e. greater $\mathrm{R}_{200}$ ), less concentrated and show an excess of fainter galaxies with respect to G clusters (Fig. 1);

- Fig. 4 highlights that higher density regions in the PPS of NG clusters extend to higher velocities in comparison to G Clusters, a possible signature from an excess of high-velocity infalling galaxies;

- The distribution of galaxy properties is systematically more mixed in the PPS of NG clusters than in G clusters (Figs. 5 and 6). This trend holds in both luminosity regimes, but it is more noticeable in F galaxies;

- Fig. 8 suggests that $\mathrm{G}$ and $\mathrm{NG}$ clusters have different accretion histories over the last $\sim 5$ Gyr. Our findings suggest that NG clusters accreted on average $\sim 10^{11} \mathrm{M}_{\odot}$ more stellar mass than $\mathrm{G}$ systems. The trend of a higher accretion rate in NG clusters is also present when comparing systems with similar $\mathrm{M}_{200}$;

These results suggest that $\mathrm{G}$ and NG clusters provide two different environments to study galaxy evolution and environmental quenching. N-body simulations show that more massive clusters sustain higher merger rates (Genel et al. 2009). In other words, massive clusters are usually formed by the interaction of two groups and/or clusters. Our sample is built upon massive clusters and hence it is expected that a large fraction of them had experienced such fusion events in the past. A higher fraction of merging events is expected in NG clusters due to the observed excess of massive systems in comparison to G groups. Such events are perturbations in the dynamical equilibrium of a cluster. From the PPS point of view, this translates into a more mixed distribution of galaxy properties in comparison to the unperturbed state. Our results point to NG clusters having more mixed distributions in the PPS. Finally, Roberts 
\& Parker (2019) show that NG clusters suffered their last major merger more recently than $\mathrm{G}$ clusters. These trends also show that NG clusters are statistically in a more unrelaxed state in comparison to $\mathrm{G}$ clusters and thus provide different environments to study galaxy evolution.

Previous works indicate that NG clusters also show higher infall rates. Here we quantify this infall rate by tracing the stellar mass in different regions of the PPS and estimate that over the last $5 \mathrm{Gyr}$ of cosmic time, NG clusters roughly accreted stellar material comparable to the stellar component of massive groups, in comparison to $\mathrm{G}$ systems. At first, approximately $2 / 3$ of the accreted mass comes from B galaxies. However, B galaxies are roughly 10 times more massive than $\mathrm{F}$ galaxies. This means that numerically there are far more $\mathrm{F}$ galaxies being accreted in comparison to B galaxies. We conclude that this is mostly due to the accretion of low-luminosity galaxies (FG), a result consistent with works that explain the non-gaussianity in the projected velocity distribution as a result of an accretion of low luminosity galaxies (e.g. de Carvalho et al. 2017; Roberts \& Parker 2019). Examining Table 3, we note that NG clusters feature shallower color gradients (i.e. slopes closer to zero) in comparison to $\mathrm{G}$ clusters. These gradients reflect a more mixed galactic population in NG systems. However, we highlight that these results are found by considering large samples of clusters to guarantee that the effects of projection along the line of sight influence both cluster classes in the same way. Costa et al. (2018) study the galaxy velocity dispersion and conclude that $\mathrm{B}$ and $\mathrm{F}$ galaxies are more distinct in $\mathrm{G}$ clusters. It follows from the mass-segregation phenomenon in clusters (Capelato et al. 1980) that relaxed systems show a clear separation between high and low mass galaxy properties, hence a more mixed population of bright and faint galaxies gives further support to the hypothesis that NG clusters are in a more unrelaxed state than $\mathrm{G}$ systems. Here we also provide further evidence that the non-gaussianity in the projected velocity distribution is connected to a higher infall rate of faint galaxies, in agreement with Roberts \& Parker (2019). However, a more detailed characterization on a one-by-one basis should be given by combining further dynamic probes such as caustic curve analysis (Gifford et al. 2013; Dawson et al. 2012), X-Ray characterization (Schuecker et al. 2001), velocity dispersion profiles (Baumgardt et al. 2019) and galaxy spatial distribution (Flin \& Krywult 2006).

\subsection{Low vs. High Mass Galaxies Quenching in Dense Environments}

One of the primary results of this work is the unequivocal trends found between $\mathrm{G}$ and $\mathrm{NG}$ clusters and the properties of the galaxy members, shown in Fig. 7. In this regard, we highlight the following points:

- The trends of age, $[\mathrm{Z} / \mathrm{H}]$, SFR and TType of B galaxies with respect to infall time show slopes closer to zero in comparison to F galaxies, indistinctly in G or NG clusters. These trends evidence how $\mathrm{B}$ and $\mathrm{F}$ galaxies are differently affected by their environment. Namely, B galaxies are more massive and hence less affected by environment-specific processes such as ram pressure stripping;

- Age and $[\mathrm{Z} / \mathrm{H}]$ have very similar relations with infall time, reinforcing the closeness between these two parameters. (Luminosityweighted) Age here roughly means the time since the last star formation episode and thus higher age translates to more time to stars evolve and increase the metallicity of the ISM;

- The similarity between the relations of SFR and TType sug- gests that quenching of star formation and morphological transformations may be simultaneously caused by a third process;

- The slopes of the trends with infall time regarding FG are consistently lower in NG clusters in comparison to G systems, for most of the six-parameter set here adopted to characterize member galaxy properties;

- F galaxies with time since infall lower than 4.5 Gyr in G clusters are younger, more metal-poor and show higher star formation rates than their counterparts in NG clusters;

These relations are of particular interest since the quenching time scale is pivotal to understanding environmental effects in galaxy evolution (Paccagnella et al. 2016). Galaxies moving within and/or towards the cluster eventually reach a threshold density that triggers efficient ram pressure stripping, removing the gas component of low mass galaxies in a short time scale $(\leq 1 \mathrm{Gyr}$, Roediger \& Brüggen 2007) quickly quenching star formation (R19). However, the trends for high mass galaxies seems to be quite different. The flatter relations between $[\mathrm{Z} / \mathrm{H}]$, SFR or TType and $t_{\text {inf }}$ for $B$ galaxies in comparison to $\mathrm{F}$ ones indicate that massive (and hence more luminous) galaxies are less affected by the environment, indistinctly in $\mathrm{G}$ or NG clusters. This result is in agreement with massive galaxies being quenched mostly due to internal mechanisms such as AGN and stellar feedback (Larson 1974). Various works debate the relation between galaxy star formation quenching and morphological transformation (Martig et al. 2009; Schawinski et al. 2014; Kelkar et al. 2019). The trends found in Fig. 7 support the close SFR morphology relation and extends it also to age and [Z/H], providing further insight into how stellar population parameters reflect galaxy evolution.

Additionally, in the faint regime, we also note striking differences between $\mathrm{G}$ and NG clusters, suggesting once more that bright galaxies are likely quenched upon internal processes. We find that galaxies with $2.5<\mathrm{t}_{\text {inf }}<4.5 \mathrm{Gyr}$ in NG systems are older and more metal-rich than their counterparts in $\mathrm{G}$ systems. This corresponds roughly to PNZs 4 to 6 , which are characterized by a mean $\left|\mathrm{V}_{\mathrm{LOS}}\right| / \sigma \sim 1.2 \pm 0.3 \mathrm{~km} \mathrm{~s}^{-1}$. A higher $\left|\mathrm{V}_{\mathrm{LOS}}\right| / \sigma$ is closely connected to infalling galaxies. Namely, galaxies with higher velocities are those first entering the cluster. The trends we find hence indicate that galaxies infalling in NG clusters are older and more metal-rich than in $\mathrm{G}$ systems. These results are in agreement with $\mathrm{dC} 17$, which find similar trends when comparing radial profiles of galaxy properties in $\mathrm{G}$ and NG clusters. Furthermore, despite our analysis being limited to $\mathrm{R}_{200}$, $\mathrm{dC} 17$ show that these differences extend until $2 \mathrm{R}_{200}$. These results are in agreement with a scenario where galaxies infalling into NG clusters have been pre-processed (P19) in comparison to those in G clusters. This is reinforced by the results found for SFR, TType and color gradient, namely we find galaxies with lower SFR and TType in NG systems. Also, faint galaxies in NG clusters show shallower color gradients than faint galaxies in $\mathrm{G}$ systems, indicating that galaxies entering NG clusters already started a transformation towards an elliptical morphology. Complementary to the results presented in this paper, de Carvalho et al. (2019) show that faint spiral galaxies are the ones that suffer major environmental effects.

This paper is the fourth of a series where we investigate how the gaussianity of the velocity distribution of the galaxy members in a cluster is of paramount importance for the studying of how galaxy evolution is affected by the environment. NG clusters exhibit a higher infall rate when contrasted to the $\mathrm{G}$ ones. Previous works have suggested this trend, here we quantify it for the first time. Also, based on the PPS analysis, we show that galaxies with high 
$\left|\mathrm{V}_{\mathrm{LOS}}\right| / \sigma$ belonging to NG clusters are older and more metalrich than the ones in $\mathrm{G}$ systems (even for $\mathrm{R}<\mathrm{R}_{200}$ ). This is a clear indication that these galaxies infalling into NG systems were preprocessed from the chemical evolution standpoint. Examining the trends of Age, $[\mathrm{Z} / \mathrm{H}], \mathrm{SFR}, \mathrm{M}_{\text {stellar }}$, TType and $\nabla(\mathrm{g}-\mathrm{i})$, with the infall time, for faint galaxies in NG versus $\mathrm{G}$, confirms that such a distinction in velocity distribution is not an artifact of the methods employed to measure it.

\section{ACKNOWLEDGEMENTS}

We would like to thank the anonymous referee for the comments and suggestions which contributed to greatly improving this article. RRdC and VMS thank T. S. Golçalves for fruitful discussions on this topic. VMS acknowledges the CAPES scholarship through the grants 88887.508643/2020-00 and 88882.444468/2019-01. RRdC aknowledges the financial support from FAPESP through the grant \#2014/11156-4. TFL acknowledges financial support from FAPESP (2018/02626-8) and CNPq (306163/2019-5). IF acknowledges support from the Spanish Ministry of Science, Innovation and Universities (MCIU), through grant PID2019-104788GB-I00. ALBR thanks the support of CNPq, grant 311932/2017-7. S.B.R. acknowledges support from Conselho Nacional de Desenvolvimento Científico e Tecnológico - CNPq. This work was made possible thanks to a number of open-source software packages: AstroPy (Astropy Collaboration et al. 2018), Matplotib (Barrett et al. 2005), NumPy (van der Walt et al. 2011), Pandas (McKinney et al. 2010) and SciPy (Virtanen et al. 2020).

\section{DATA AVAILABILITY}

The data used in this manuscript will be made available under request.

\section{REFERENCES}

Abadi M. G., Moore B., Bower R. G., 1999, MNRAS, 308, 947

Adelman-McCarthy J. K., et al., 2007, ApJS, 172, 634

Albareti F. D., et al., 2017, ApJS, 233, 25

Anderson T. W., Darling D. A., 1952, The annals of mathematical statistics, pp 193-212

Angthopo J., Ferreras I., Silk J., 2019, MNRAS, 488, L99

Astropy Collaboration et al., 2018, AJ, 156, 123

Balogh M. L., Navarro J. F., Morris S. L., 2000, ApJ, 540, 113

Barrett P., Hunter J., Miller J. T., Hsu J. C., Greenfield P., 2005, in Shopbell P., Britton M., Ebert R., eds, Astronomical Society of the Pacific Conference Series Vol. 347, Astronomical Data Analysis Software and Systems XIV. p. 91

Baumgardt H., Hilker M., Sollima A., Bellini A., 2019, MNRAS, 482, 5138

Benkert T., Franke K., Pozitron D., Standish R., 2014, D005 (Free Software Foundation, Inc: 51 Franklin Street, Fifth Floor, Boston, MA 021101301 USA)

Blanton M. R., et al., 2005, AJ, 129, 2562

Bongiorno A., et al., 2016, A\&A, 588, A78

Brinchmann J., Charlot S., White S. D. M., Tremonti C., Kauffmann G., Heckman T., Brinkmann J., 2004, MNRAS, 351, 1151

Capelato H. V., Gerbal D., Salvador-Sole E., Mathez G., Mazure A., Sol H., 1980, ApJ, 241, 521

Choi Y.-Y., Han D.-H., Kim S. S., 2010, Journal of Korean Astronomical Society, 43, 191

Cid Fernandes R., Mateus A., Sodré L., Stasińska G., Gomes J. M., 2005, MNRAS, 358, 363
Costa A. P., Ribeiro A. L. B., de Carvalho R. R., 2018, MNRAS, 473, L31

Cox T. J., Jonsson P., Somerville R. S., Primack J. R., Dekel A., 2008, MNRAS, 384, 386

Dawson W. A., et al., 2012, ApJ, 747, L42

De Helguero Roma D. D. F., 1904, Biometrika, 3, 84

Dekel A., Silk J., 1986, ApJ, 303, 39

Domínguez Sánchez H., Huertas-Company M., Bernardi M., Tuccillo D., Fischer J. L., 2018, MNRAS, 476, 3661

Dressler A., 1980, ApJ, 236, 351

Dressler A., Shectman S. A., 1988, AJ, 95, 985

Engmann S., Cousineau D., 2011, Journal of applied quantitative methods, 6,1

Fadda D., Girardi M., Giuricin G., Mardirossian F., Mezzetti M., 1996, ApJ, 473,670

Feigelson E. D., Babu G. J., 2012, Modern Statistical Methods for Astronomy

Flin P., Krywult J., 2006, A\&A, 450, 9

Fujita Y., 2004, PASJ, 56, 29

Gehan E. A., 1965, Biometrika, 52, 203

Genel S., Genzel R., Bouché N., Naab T., Sternberg A., 2009, ApJ, 701, 2002

Gifford D., Miller C., Kern N., 2013, The Astrophysical Journal, 773, 116

Girardi M., Escalera E., Fadda D., Giuricin G., Mardirossian F., Mezzetti M., 1997, ApJ, 482, 41

Gunn J. E., Gott J. Richard I., 1972, ApJ, 176, 1

Hansen S. H., Egli D., Hollenstein L., Salzmann C., 2005, New Astron., 10, 379

Johnston K. V., Sigurdsson S., Hernquist L., 1999, MNRAS, 302, 771

Kelkar K., Gray M. E., Aragón-Salamanca A., Rudnick G., Jaffé Y. L., Jablonka P., Moustakas J., Milvang-Jensen B., 2019, MNRAS, 486, 868

Larson R. B., 1974, MNRAS, 169, 229

Larson R. B., Tinsley B. M., Caldwell C. N., 1980, ApJ, 237, 692

Le Cam L., Yang G. L., 2012, Asymptotics in statistics: some basic concepts. Springer Science \& Business Media

Lopes P. A. A., de Carvalho R. R., Kohl-Moreira J. L., Jones C., 2009a, MNRAS, 392, 135

Lopes P. A. A., de Carvalho R. R., Kohl-Moreira J. L., Jones C., 2009b, MNRAS, 399, 2201

Lynden-Bell D., 1967, MNRAS, 136, 101

Mahajan S., 2013, MNRAS, 431, L117

Mahajan S., Mamon G. A., Raychaudhury S., 2011, MNRAS, 416, 2882

Martig M., Bournaud F., Teyssier R., Dekel A., 2009, ApJ, 707, 250

McKinney W., et al., 2010, in Proceedings of the 9th Python in Science Conference. pp 51-56

Merrall T. E., Henriksen R. N., 2003, The Astrophysical Journal, 595, 43

Neistein E., van den Bosch F. C., Dekel A., 2006, MNRAS, 372, 933

Ogorodnikov K. F., 1957, Azh, 34, 770

Oman K. A., Hudson M. J., Behroozi P. S., 2013, MNRAS, 431, 2307

Oman K. A., Bahé Y. M., Healy J., Hess K. M., Hudson M. J., Verheijen M. A. W., 2020, MNRAS,

Paccagnella A., et al., 2016, ApJ, 816, L25

Park C., Choi Y.-Y., 2005, ApJ, 635, L29

Pasquali A., Smith R., Gallazzi A., De Lucia G., Zibetti S., Hirschmann M., Yi S. K., 2019, MNRAS, 484, 1702

Peng Y.-j., et al., 2010, ApJ, 721, 193

Read J. I., Wilkinson M. I., Evans N. W., Gilmore G., Kleyna J. T., 2006, MNRAS, 366, 429

Reynolds D. A., 2009, Encyclopedia of biometrics, 741

Rhee J., Smith R., Choi H., Yi S. K., Jaffé Y., Candlish G., Sánchez-Jánssen R., 2017, ApJ, 843, 128

Rhee J., Smith R., Choi H., Contini E., Jung S. L., Han S., Yi S. K., 2020, ApJS, 247, 45

Ribeiro A. L. B., Lopes P. A. A., Trevisan M., 2010, MNRAS, 409, L124

Ribeiro A. L. B., de Carvalho R. R., Trevisan M., Capelato H. V., La Barbera F., Lopes P. A. A., Schilling A. C., 2013, MNRAS, 434, 784

Roberts M. S., Haynes M. P., 1994, ARA\&A, 32, 115

Roberts I. D., Parker L. C., 2017, MNRAS, 467, 3268

Roberts I. D., Parker L. C., 2019, MNRAS, 490, 773 
Roberts I. D., Parker L. C., Brown T., Joshi G. D., Hlavacek-Larrondo J., Wadsley J., 2019, ApJ, 873, 42

Roediger E., Brüggen M., 2007, MNRAS, 380, 1399

Sánchez-Blázquez P., et al., 2006, MNRAS, 371, 703

Sarron F., Adami C., Durret F., Laigle C., 2019, A\&A, 632, A49

Sazonova E., et al., 2020, ApJ, 899, 85

Schawinski K., et al., 2014, MNRAS, 440, 889

Schilling M. F., Watkins A. E., Watkins W., 2002, The American Statistician, 56,223

Schuecker P., Böhringer H., Reiprich T. H., Feretti L., 2001, A\&A, 378, 408

Silverman B. W., 1986, Density estimation for statistics and data analysis

Springel V., Hernquist L., 2005, ApJ, 622, L9

Teyssier R., Chapon D., Bournaud F., 2010, ApJ, 720, L149

Trevisan M., Ferreras I., de La Rosa I. G., La Barbera F., de Carvalho R. R., 2012, ApJ, 752, L27

Trussler J., Maiolino R., Maraston C., Peng Y., Thomas D., Goddard D., Lian J., 2020, MNRAS, 491, 5406

Virtanen P., et al., 2020, scipy/scipy: SciPy 1.5.3, doi: $10.5281 /$ zenodo. 595738

Wetzel A. R., Tinker J. L., Conroy C., 2012a, Monthly Notices of the Royal Astronomical Society, 424, 232

Wetzel A. R., Tinker J. L., Conroy C., 2012b, MNRAS, 424, 232

Wetzel A. R., Tinker J. L., Conroy C., van den Bosch F. C., 2013, MNRAS, 432, 336

Wojtak R., Łokas E. L., 2010, MNRAS, 408, 2442

Yang X., Mo H. J., van den Bosch F. C., Pasquali A., Li C., Barden M., 2007, ApJ, 671, 153

Zhang Y.-Y., Reiprich T. H., Finoguenov A., Hudson D. S., Sarazin C. L., 2009, ApJ, 699, 1178

Zu Y., Mandelbaum R., 2016, MNRAS, 457, 4360

de Carvalho R. R., Ribeiro A. L. B., Stalder D. H., Rosa R. R., Costa A. P., Moura T. C., 2017, AJ, 154, 96

de Carvalho R. R., Costa A. P., Moura T. C., Ribeiro A. L. B., 2019, MNRAS, 487, L86

de Vaucouleurs G., 1963, ApJS, 8, 31

van de Voort F., Bahé Y. M., Bower R. G., Correa C. A., Crain R. A., Schaye J., Theuns T., 2017, MNRAS, 466, 3460

van der Walt S., Colbert S. C., Varoquaux G., 2011, Computing in Science and Engineering, 13, 22

\section{APPENDIX A: COMPARISON BETWEEN RHEE REGIONS VS. PASQUALI'S NEW ZONES}

There are different ways to separate galaxies in projected phase space. In this work we slice the PPS in PNZs (Pasquali et al. 2019), which divide galaxies according to their infall time. This choice, however, is not unique. The definition of PNZs is restricted to cluster-centric distances within $R_{200}$. To explore the outer areas of clusters, we also adopt the "Rhee Regions" (R17) as a second way of dividing the PPS tracing galaxies infall time. This second way of discretizing the PPS is based on a probabilistic approach. Therefore, there is no direct relation between Rhee Regions and PNZs. In Fig. A1 we show the relation between Rhee Region and galaxy properties. Examining Figs 7 and A1 we find that our analysis with Rhee Regions or PNZs lead to quite similar global trends. Similarly to the PNZs case, we find that the most striking differences are in the faint regime. Additionally, the similarity extends to the trends we observe. From panels (a) and (b), we note that $\mathrm{F}$ galaxies in regions $\mathrm{D}$ and $\mathrm{C}$ of $\mathrm{NG}$ clusters are older and more metal-rich than its counterpart in $\mathrm{G}$ systems. In panel (c), we find that there are no significant differences between stellar mass of $F$ galaxies in $G$ or NG systems. Regarding SFR (panel d), comparison shows that faint galaxies in regions $\mathrm{D}, \mathrm{C}$ and $\mathrm{B}$ of $\mathrm{G}$ clusters are more star forming than those in NG systems. This is also related to morphology (panel e), for which $\mathrm{F}$ galaxies in regions $\mathrm{D}$ and $\mathrm{C}$ of NG clusters have higher TType values (closer to elliptical). Finally, in panel (f) we note that $\mathrm{F}$ galaxies in NG clusters have shallower (closer to 0 ) color gradients than in G systems. However, We highlight that there are less Rhee Regions than PNZs, which leads to a more "curvy" shaped curves due to the adopted procedure. This paper performs most of the analysis using the PNZs and, when the $\mathrm{R}_{200}$ limitation plays a major role (Section 7, for instance), we switch to the alternative scheme.

This paper has been typeset from a $\mathrm{T}_{\mathrm{E}} \mathrm{X} / \mathrm{L} \mathrm{T} \mathrm{E} \mathrm{X}$ file prepared by the author. 

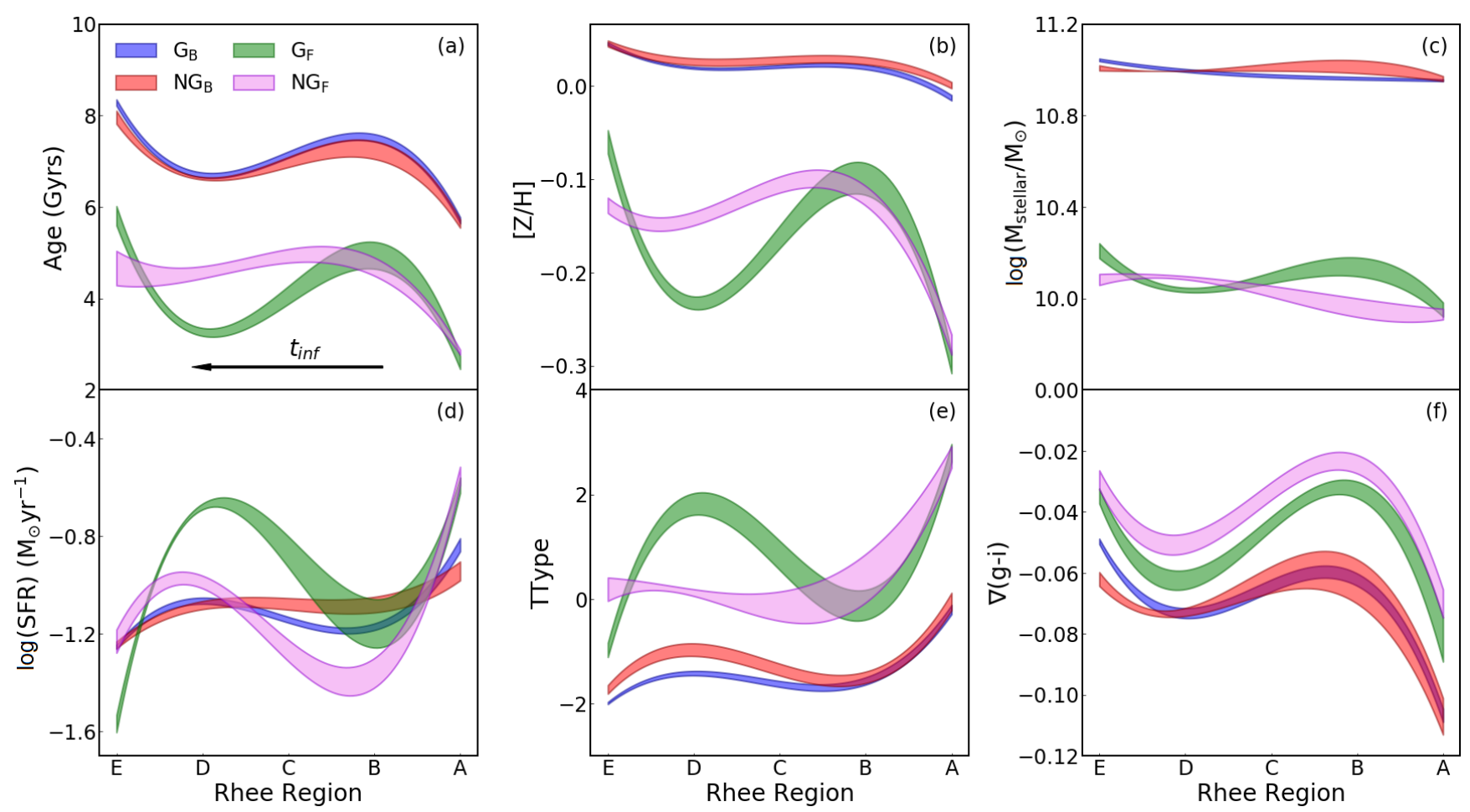

Figure A1. Same as Fig. 7, but using Rhee Regions (instead of PNZ) in order to map the location on the PPS and tinf. Colors follow the same schema of Fig. 7. The curves and their width represent a spline fitting and the associated variance, respectively. 